\title{
Factors Controlling the Input-Output Relationship of Spherical Bushy Cells in the Gerbil Cochlear Nucleus
}

\author{
Thomas Kuenzel, J. Gerard G. Borst, and Marcel van der Heijden \\ Department of Neuroscience, Erasmus Medical Center, NL-3000 CA Rotterdam, The Netherlands
}

Despite the presence of large endbulb inputs, the spherical bushy cells (SBCs) of the rostral anteroventral cochlear nucleus do not function as simple auditory relays. We used the good signal-to-noise ratio of juxtacellular recordings to dissect the intrinsic and network mechanisms controlling the input- output relationship of SBCs in anesthetized gerbils. The SBCs generally operated close to action potential (AP) threshold and showed no evidence for synaptic depression, suggesting that the endbulbs of Held have low release probability in vivo. Analysis of the complex waveforms suggested that in the absence of auditory stimulation, postsynaptic spike depression and stochastic fluctuations in EPSP size were the main factors determining jitter and reliability of the endbulb synapse. During auditory stimulation, progressively larger EPSPs were needed to trigger APs at increasing sound intensities. Simulations suggested hyperpolarizing inhibition could explain the observed decrease in EPSP efficacy. Synaptic inhibition showed a delayed onset and generally had a higher threshold than excitatory inputs, but otherwise inhibition and excitation showed mostly overlapping frequency-response areas. The recruitment of synaptic inhibition caused postsynaptic spikes to be preferentially triggered by well-timed, large EPSPs, resulting in improved phase locking despite more variable EPSP-AP latencies. Our results suggest that the lack of synaptic depression, caused by low release probability, and the apparent absence of sound-evoked synaptic inhibition at low sound intensity maximize sensitivity of SBCs. At higher sound intensities, the recruitment of synaptic inhibition constrains their firing rate and optimizes their temporal precision.

\section{Introduction}

Spherical bushy cells (SBCs) are the principal neurons of the anteroventral cochlear nucleus (AVCN). Their main projection targets are the lateral and medial superior olive (MSO), which are both involved in sound localization (Cant and Benson, 2003). An $\mathrm{SBC}$ is contacted by one to four giant synaptic terminals, the endbulbs of Held (Cant and Morest, 1979; Ryugo and Sento, 1991; Cao et al., 2010). These inputs can cover a large part of their soma (Sento and Ryugo, 1989; Liberman, 1991), and a single endbulb input can already be sufficiently strong to trigger an action potential (AP) in the rat SBC (Isaacson and Walmsley, 1995). Despite the presence of large endbulb inputs, in vivo studies have shown that the SBCs do not have a simple relay function, since the transmission between the auditory nerve and the SBC can show frequent failures (Kopp-Scheinpflug et al., 2002; Englitz et al., 2009). The cause of these failures is still debated.

During high-frequency stimulation, the rodent endbulb synapse shows synaptic depression in slice recordings (Bellingham and Walmsley, 1999; Oleskevich et al., 2000; Wang and Manis, 2008; Yang and Xu-Friedman, 2008; Cao et al., 2010; Chanda and Xu-Friedman, 2010a,b). In the depressed state, multiple endbulb inputs may be needed to trigger a spike in the SBC (Yang and

Received 0ct. 17, 2010; revised Jan. 17, 2011; accepted Jan. 24, 2011.

This work was supported by Neuro-BSIK Grant BSIK 03053 (SenterNovem, The Netherlands). We thank L. Carney for advice on the stereotactical orientation of the recording electrode and S. W. F. Meenderink for helpful comments on this manuscript.

Correspondence should be addressed to Dr. Thomas Kuenzel, Department of Neuroscience, Erasmus Medical Center, P.0. Box 2040, NL-3000 CA Rotterdam, The Netherlands. E-mail: t.kunzel@erasmusmc.nl.

DOI:10.1523/JNEUROSCI.5433-10.2011

Copyright $\odot 2011$ the authors $\quad 0270-6474 / 11 / 314260-14 \$ 15.00 / 0$
Xu-Friedman, 2009). This convergence of multiple inputs onto the SBCs is thought to increase the temporal accuracy of the auditory signal compared with the auditory nerve (Rothman et al., 1993; Joris et al., 1994a,b; Paolini et al., 2001; Xu-Friedman and Regehr, 2005a,b; Dehmel et al., 2010). In addition, SBCs receive mainly glycinergic but also GABAergic inhibitory inputs (Wickesberg and Oertel, 1990; Zhang and Oertel, 1993; Juiz et al., 1996), which have a strong impact on the output of the SBC in vivo (Winter and Palmer, 1990; Caspary et al., 1994; Paolini and Clark, 1998; Kopp-Scheinpflug et al., 2002).

Few efforts have been made to reconcile the view obtained from slice recordings of the SBC as a cell that receives suprathreshold, phasic, precise inputs, with the in vivo view of the $\mathrm{SBC}$ as a cell that is strongly influenced by inhibitory inputs, and which relies on multiple, coincident excitatory inputs for precise timing. An advantageous circumstance is that, because of the large size of the endbulb inputs, in vivo recordings from SBCs are characterized by complex extracellular waveforms (Pfeiffer, 1966; KoppScheinpflug et al., 2002; Englitz et al., 2009). The complex waveform consists of a prespike, which presumably reflects the AP of a nearby endbulb of Held, and an extracellularly recorded EPSP, which may or may not trigger an AP (Pfeiffer, 1966; Typlt et al., 2010). Lorteije et al. (2009) showed that similar waveforms recorded from the calyx of Held synapse can be used to estimate both the strength of synaptic transmission and postsynaptic excitability. Here, we analyze juxtacellular recordings from SBCs in the rostral AVCN of anesthetized adult gerbils and show that the complex waveforms can also be used to quantify synaptic inhibition, allowing identification of the different factors governing precision and reliability of the SBC in vivo. 


\section{Materials and Methods}

A total of 29 female Mongolian gerbils (Meriones unguiculatus) weighing $59 \pm 6 \mathrm{~g}$ were used. All animal procedures were in accordance with the European Communities Council Directive (86/609/EEC) and approved by a Dutch animal ethics committee.

Surgical approach and recording technique. Animals were anesthetized with an intraperitoneal injection of ketamine ( $80 \mu \mathrm{g} / \mathrm{g}$ body weight) and xylazine (Rompun, $12 \mu \mathrm{g} / \mathrm{g}$ body weight). Anesthesia was maintained with subcutaneous injections of one-third of this dose every hour or when needed. A head mount was glued to the exposed skull. A craniotomy was performed at a location delimited by the superior semicircular canal within the superior anterior chamber of the bulla, as described by Frisina et al. (1982). The pinna on the same side of the head was carefully removed to allow unrestricted placement of the custom-built speaker probe over the bony ear canal. The probe combined an earphone speaker (Shure SCL2) and a microphone for in situ calibration (Type 40AG; G.R.A.S.).

Animals were mounted on a custom-built platform positioned on a vibration-isolated table in a double-walled soundproof chamber (Acoustair B.V.). For recordings in the rostral AVCN, the path of the electrode was as follows: projected on the horizontal plane, it was angled at $45^{\circ}$ from the rostrocaudal axis toward rostral; projected on the midsagittal plane, it was angled at $20-25^{\circ}$ from the rostrocaudal axis toward rostral. To improve visibility, a vertically aligned electrode was used, and the platform was rotated to meet these angles. Body temperature was maintained at $37^{\circ} \mathrm{C}$ with a heating pad. The reference electrode was placed in muscle tissue.

Recordings were performed with $1.5 \mathrm{~mm}$ outer diameter glass micropipettes (Hilgenberg) containing a K-gluconate-based internal solution (in mM: $126 \mathrm{~K}$-gluconate, $20 \mathrm{KCl}, 10 \mathrm{Na}_{2}$-phosphocreatine, $4 \mathrm{Mg}$ ATP, $0.3 \mathrm{Na}_{2}$-GTP, 0.5 EGTA, 10 HEPES; $\mathrm{pH}$ adjusted to 7.2 with $\mathrm{KOH}$; $310 \mathrm{mOsmol}$ ). For electrode track marking, the internal solution contained $0.5 \%$ biocytin. Pipettes were advanced with high positive pressure (300 mbar) through nonauditory brain tissue. On entering the auditory brainstem (as determined by multiunit responses to tonal search stimuli), pressure was reduced to 30 mbar. Electrodes were advanced in $1 \mu \mathrm{m}$ steps until single-unit responses were encountered, characterized by signal amplitudes $>2 \mathrm{mV}$ and at least a fivefold increase in pipette resistance. Then pressure was equalized, or slight negative pressure $(-5$ mbar) was applied. Waveforms were monitored on-line during recordings. Units with simple biphasic or indeterminate waveforms were normally excluded. Thus, our dataset is strongly biased toward bushy cell units showing complex waveforms and primary-like peristimulus histograms.

Voltage signals were amplified and bandpass filtered (3 Hz to $16 \mathrm{kHz}$ ) with a MultiClamp 700B intracellular amplifier (Molecular Devices) and digitized with a Digidata 1322A (Molecular Devices) at a sampling rate of $100 \mathrm{kHz}$. Full waveforms were stored for off-line analysis using the Clampex software (PClamp 8.0; Molecular Devices) running on a Windows personal computer.

Acoustic stimulation. Stimulus waveforms were generated at variable sampling rates using MATLAB (The MathWorks), transferred to a digital-to-analog converter [PD1; Tucker-Davis Technologies (TDT)], attenuated (PA4; TDT), and presented to the ipsilateral ear via earphone (SHURE, see above; HB5, TDT) for closed-field stimulation. The stimulus consisted of $50 \mathrm{~ms}$ pure tones (2-4 $\mathrm{ms} \cos ^{2}$ ramps included) presented every $400 \mathrm{~ms}$. After 10 repetitions, the frequency was generally increased by one-fourth octave, spanning a total of 18-23 frequencies. The same tone sequence was then repeated at a different sound pressure level (SPL). Using $10 \mathrm{~dB}$ steps, six to nine SPLs were used, depending on the threshold of the unit. Together, the frequency $\times$ SPL grid yielded the frequency-response area of the unit. Signals were compensated for the acoustic transfer function of the probe tube (amplitude and phase) measured in situ.

Histological verification of recording sites. Routinely, one penetration per animal was performed with $0.5 \%$ biocytin in the internal solution to mark the electrode track in the brain. At the end of the recording, biocytin was deposited by applying positive pressure (300 mbar). Animals were killed with pentobarbital (100 $\mu \mathrm{g} / \mathrm{g}$ body weight) and perfused transcardially with $0.7 \% \mathrm{NaCl}$ solution followed by $4 \%$ paraformaldehyde (PFA). Brains were postfixed in 4\% PFA overnight, stored in $10 \%$ sucrose, embedded in gelatin blocks, and processed further. Brainstems were cut into $80 \mu \mathrm{m}$ sections on a microtome. Standard histological techniques were used to visualize biocytin in the brain tissue (Vectastain kit; Vector Laboratories) and counterstain cell bodies (thionine staining).

Data analysis. All off-line analysis of waveforms was performed using custom software written in MATLAB (version 7.6). Routinely, two types of events could be discerned in our recordings, one composed of two signal components [named P and A by Pfeiffer (1966)] and the other composed of three components ( $\mathrm{P}, \mathrm{A}$, and $\mathrm{B})$. Throughout this text, we will refer to component $\mathrm{P}$ as prespike, to component $\mathrm{A}$ as extracellularly recorded EPSP (eEPSP), and to component $\mathrm{B}$ as extracellularly recorded action potential (eAP). Our analysis was based on two consecutive feature-extraction steps. First, short monotonically rising or falling sections of the waveform were identified as being candidate parts of nonrandom events, based on whether the voltage change significantly exceeded the baseline noise and on the existence of neighboring candidate sections. From this pool of candidate sections, complete complex events were extracted based on criteria of biological plausibility. All event types were detected by a threshold criterion for the rising flank of the eEPSP. Subsequently, three-component events (prespike, eEPSP, eAP; i.e., successful events) were distinguished from two-component events (prespike and eEPSP only; i.e., failures) by the presence of a steeply descending segment, since eAPs always had a steeper falling flank than eEPSPs. The threshold values were kept fixed for each unit but were adapted across units depending on the overall amplitude and signal-tonoise ratio of the recordings.

The prespike was identified as a local maximum in the waveform in a time window preceding the eEPSP. For three-component waveforms, the distinction between the eEPSP and eAP component was based on detection of an inflection in the rising flank. The amplitude of the eEPSP was then taken to be equal to the voltage level at which the inflection occurred. If the eEPSP was clearly separated from the eAP, its local maximum served as eEPSP amplitude. Following the identification of the eEPSP and eAP component, various metrics were extracted to describe the shape and relative timing of the complex waveform components. An important metric was eEPSP ${ }_{\max }^{\prime}$, the maximum rising slope of the eEPSP, which was used to characterize the strength of the EPSP (see Results). eEPSPs were timed based on their steepest rising slope; eAPs were timed based on their peak amplitude.

Analysis of threshold EPSP. We use a phenomenological definition of threshold as being the minimal "strength" of a postsynaptic event capable of eliciting an AP. In a scatter plot of eEPSP ${ }_{\text {max }}^{\prime}$ against the interspike interval for individual events (compare Fig. $3 f$ ), the threshold EPSP is represented by the line optimally dividing the populations of successful and failed data points. Events were binned according to eEPSP ${ }_{\max }^{\prime}$ and for every bin the ratio of failures to successful events was calculated. A Gaussian error function was fitted to this. Threshold was identified as the optimal dividing value (i.e., the locus of the $50 \%$ point of the error function).

In spontaneous recordings, a sliding-window technique was used to estimate the interval dependency of the threshold EPSP. From this, a range of intervals was chosen over which threshold EPSP showed little dependency on interval (typically 3-30 ms). Restricting the threshold estimation to this interval range, threshold elevation was then obtained by comparing the threshold values between tone responses and spontaneous recordings. To compare threshold elevation across units, threshold EPSPs were normalized using the total range of eEPSP' max $_{\text {ax }}$ values observed in driven and spontaneous events for each unit.

First-order model of intrinsic causes for failures. The interval dependency of the reduction of excitability was extracted for every unit from spontaneous events by calculating the ratio of failed and total events for binned interspike intervals. This provided an empirical estimate for the probability of a given event to fail as a function of "postspike time," the time since the last successful event $\left(\mathrm{P}_{\text {fail/ISI }}\right)$. The complement of this probability function $\left(1-\mathrm{P}_{\text {fail/ISI }}\right.$; i.e., the success rate) served as weight 
values for individual events recorded under sound stimulation. The sum of the weight values for a given stimulus condition corresponds to the predicted output rate.

Biophysical models of input interaction in SBCs. We constructed a model of SBCs using the NEURON simulation environment (Hines and Carnevale, 1997). We based our model on published models (Rothman et al., 1993; Rothman and Manis, 2003), using similar total conductance and basic membrane properties, and used a spatial multicompartment model consisting of a soma, an AP initiation site ("axon hillock"), and a passive stretch of axon. Basic parameters were as follows: capacitance, $20.1 \mathrm{pF}$; input resistance (at rest), 69.2 $\mathrm{M} \Omega$; membrane potential at rest, $-65.1 \mathrm{mV}$. The voltage-activated sodium conductance (1000 nS) was situated in the axon hillock segment of the model. As in the published SBC model, a significant low-voltage-activated potassium (200 nS) conductance and a hyperpolarization-activated conductance ( $40 \mathrm{nS} ; E_{\mathrm{rev}}=$ $-43 \mathrm{mV}$ ) were included. High-voltage-activated potassium conductance $(175 \mathrm{nS})$ and passive leak conductance $\left(14.5 \mathrm{nS}, E_{\text {rev }}=-65 \mathrm{mV}\right)$ were added. No dendritic compartment was included.

A simple conductance-change-based model of an excitatory synapse ( $50 \mathrm{nS} ; 0.3 \mathrm{~ms}$ tau; $E_{\text {rev }}=0 \mathrm{mV}$ ) was attached to the somatic segment. The conductance of the excitatory synapse was chosen to be suprathreshold when activated at rest. In additional simulations, we varied the excitatory synaptic conductance (between 0 and $65 \mathrm{nS}$ ) to simulate the stochastic variability of synaptic events observed in our in vivo recordings. This manipulation allowed measurement of the changes in the threshold EPSP of the model (as the eEPSP max just strong enough to trigger an AP; see below).

In addition to the excitatory synapse model, a simulated currentclamp electrode and an inhibitory conductance ( $50 \mathrm{nS} ; E_{\text {rev }}=-80 \mathrm{mV}$ ) were included to explore interaction with the excitatory input. Since the amount of inhibitory conductance is unlikely to reflect the in vivo situation, we also simulated a weaker inhibitory synaptic input (10 ms tau; 10 $\mathrm{nS} ; E_{\mathrm{rev}}=-80 \mathrm{mV}$ ) interacting with the excitatory input. The inhibitory input was trailing by $1 \mathrm{~ms}$, so that a single pair of excitatory and inhibitory inputs did not show significant interaction. Repetitive stimulation of the inputs at intervals shorter than the time constant of the inhibitory synapse model (but significantly longer than the time constant of the excitatory synapse model) caused a summation of the inhibitory conductance which could affect subsequent excitatory inputs.

To examine the sensitivity of the model to changes in low- and highvoltage-activated potassium as well as $I_{\mathrm{h}}$ conductances, calculations were repeated while varying one conductance in 100 steps. Conductance ranges were taken from Cao et al. (2007), who determined these values for small SBCs of mice (low-voltage-activated potassium, 40-200 nS; high-voltage-activated potassium, $40-174 \mathrm{nS} ; I_{\mathrm{h}}, 14-56 \mathrm{nS}$ ). In addition, $I_{\mathrm{h}}$ and low-voltage-activated potassium conductances were covaried in the same ranges as above. The results of these calculations are shown in supplemental Figures 4 and 5 (available at www.jneurosci.org as supplemental material).

We used the first derivative of the simulated whole-cell potential as an approximation of our juxtacellular in vivo recordings (Lorteije et al., 2009). In fact, the time derivative resembled the waveforms seen in juxtacellular recordings from SBCs in the intact AVCN in many aspects (with the exception of a prespike). For a more in-depth model of the juxtacellular recording situation, compare the work of Lorteije et al. (2009). Consistent with the analysis of our in vivo data, we used the maximum slope of the simulated eEPSP component to estimate the strength of the synaptic event, which corresponds to the maximum of the second derivative of the simulated whole-cell potential (iEPSP").

Recovery models of spike depression and synaptic facilitation. Recovery models of spike depression and synaptic facilitation were fitted to data from spontaneous recordings (Varela et al., 1997; Lorteije et al., 2009). Briefly, a time-dependent state variable was calculated, which was cumulatively decreased (depression) or increased (facilitation) by a given amount whenever a spike or event occurred. The state variable relaxed back to its ground state value after an exponential function. At measured spike or event times, the instantaneous eAP amplitude was predicted as maximum eAP amplitude $\times$ state variable. Analogously, predicted eEPSP amplitude was initial eEPSP amplitude $\times(1+$ state variable $)$. Pre- dicted amplitudes were compared with measured amplitudes, and model parameters (time constants and magnitudes of the exponential functions; values for maximum eEPSP'/eAP) were fitted to the data by minimizing the sum of squared errors. A total of $6 \mathrm{~s}$ of spontaneous recording was used per unit to estimate time constants. The validity of the fitting parameters was checked by plotting the predicted and measured event metrics against the event interval and by calculating the mean metrics in logarithmic interval bins. Coefficients of correlation (MATLAB function corrcoef.m) for these binned averages were calculated for further validation of the model parameters. Only fitted parameters for units for which a significant $(p<0.05)$ correlation was found were accepted.

Simulating the effect of a strict EPSP threshold on phase locking. The effect of a strictly imposed EPSP threshold on phase locking was analyzed from sets of events pooled from repeated presentations of a single stimulus frequency. All events, whether successful or not, were pooled and from this pool those events were selected whose eEPSP max $_{\text {ax }}$ exceeded a given threshold. Vector strengths calculated from this restricted set were compared to the vector strength calculated from the total pool of events. The increase in vector strength was plotted against the simulated threshold EPSP. To check for the effect of reduced spike counts, the same amount of events was also randomly selected from the pool of events, and this random selection was repeated 1000 times for every condition. Mean and SD of resulting vector strength values was calculated.

Estimating error bars for measured vector strength values. To estimate the uncertainty in vector strength values, we used a bootstrapping method. Vector strength $R$ was computed for a large number of random event trains drawn from Gaussian distributions of arrival times within the stimulus cycle, using many different widths and event numbers $N$. For each subset of fixed width and event count, the interquartile range of $R$ values was determined, yielding a look-up table that linked a given pair $(R, N)$ to the interquartile range of the corresponding subset of event trains.

Other statistical methods. Unless noted otherwise, data are expressed as mean \pm 1 SD. Statistical difference was determined with a paired, onetailed $t$ test. To compare relative changes of waveform metrics (i.e., interval-dependent eAP amplitude) between units, metrics were sometimes normalized by dividing values for every event by the maximum value occurring for that metric in a given unit.

\section{Results}

We analyzed recordings from 84 units in the rostral AVCN of anesthetized gerbils. The units showed a clear bias toward low and mid characteristic frequencies (CFs) $(1.5 \pm 0.8 \mathrm{kHz} ; 48 \mathrm{~Hz} \leq$ $\mathrm{CF} \leq 5662 \mathrm{~Hz}$ ), consistent with their predominant location in the rostral AVCN (Englitz et al., 2009). The mean minimal threshold ( $17 \pm 13 \mathrm{~dB}$ SPL; minimum, $-5 \mathrm{~dB}$ SPL; maximum, $50 \mathrm{~dB}$ SPL) was compatible with the low CFs. Spontaneous firing rates were moderate to high ( $44 \pm 29$ spikes/s; range, $0-102$ spikes/s).

Fifty percent (42 of 84 ) of the units showed discernible prespikes in addition to a complex waveform, qualifying them as SBCs (Englitz et al., 2009). Based on this identification, we selected 39 SBCs for further analysis, discarding three units for which waveform analysis was not feasible (see Materials and Methods). All of these 39 SBCs showed primary-like poststimulus time histograms. The CFs of the SBCs were not different from the 42 non-prespike units (SBCs, $1592 \pm 918 \mathrm{~Hz} ; n=39 ; p=$ $0.37)$. The $39 \mathrm{SBCs}$ had significantly higher spontaneous firing rates (56 \pm 23 spikes/s; $n=39 ; p=0.0004$ ) and lower minimal thresholds ( $11 \pm 11 \mathrm{~dB} \mathrm{SPL} ; n=39 ; p=0.0003)$ compared with non-prespike units. In all (15 of 15) brains that were prepared for histological procedures after the recordings, the rostral part of the AVCN anterior of the nerve root showed either biocytin bulk labeling of the electrode tract or individual neurons filled with biocytin (Fig. 1a).

Based on the characteristics of our prespike units, we assume that they predominantly represent a cell type corresponding to 
a

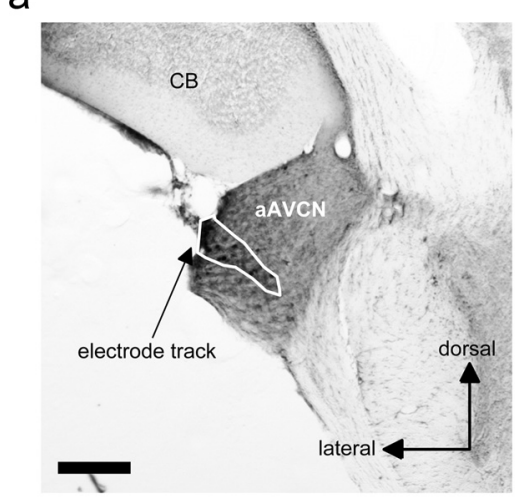

e

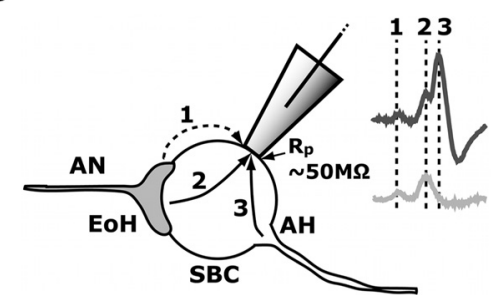

b

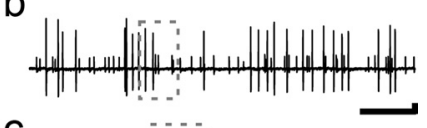

C

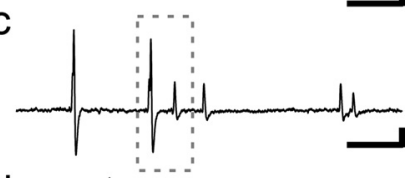

d

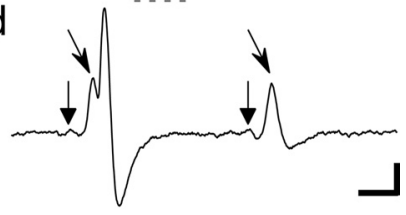

f

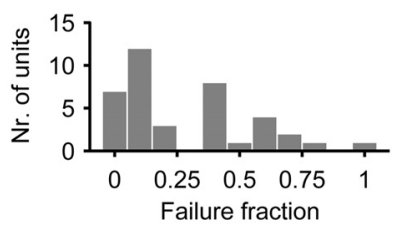

Figure 1. $\quad \boldsymbol{a}$, Frontal section with biocytin-labeled electrode track (white outline) in the rostral AVCN. In all 15 tested animals, biocytin labeling was found anterior of the nerve root area in the AVCN. Scale bar, $250 \mu \mathrm{m}$. CB, Cerebellum; aAVCN, anterior AVCN. (animal: RG09171) $\boldsymbol{b}$ - d, Juxtacellular waveforms of spontaneous events at different time scales. Scale bars: $y$-axis, $1 \mathrm{mV} ; x$-axis, 100/10/1 ms. (unit: RG09171u7) e, Schematic of the interpretation of the waveform components. The distance between electrode tip and synaptic terminal is exaggerated for clarity. A marked prespike component likely indicates close proximity between electrode tip and synaptic terminal. AN, Auditory nerve; EoH, endbulb of Held synaptic terminal; $R_{p}$, seal resistance; $A H$, axon hillock. $f$, Distribution of mean failure probability during spontaneous activity of 39 units.

\section{Synaptic failures}

A basic metric derived from the recordings is the failure rate, the fraction of failures (events not comprising an eAP) among the total number of events. Most SBCs have nonzero failure rates in the $\mathrm{ab}$ sence of auditory stimulation (Englitz et al., 2009). The histogram in Figure $1 f$ shows the distribution of spontaneous failure rates measured in the 39 SBCs. On average, $29 \pm 26 \%$ of all spontaneous synaptic events were failures. This percentage did not differ between the 39 SBCs and the 42 AVCN units without discernable prespikes $(30 \pm 29 \% ; p=0.72)$.

The high failure rates of spontaneous synaptic events generated a marked difference between the input (all eEPSPs) and the output (eAPs) of the SBC in spontaneous recordings. We will show later that this contrast became even more prominent after auditory stimulation. It is therefore important to identify the underlying causes of transmission failures. Spontaneous recordings offer an optimal framework for this analysis, because in the absence of a stimulus, the interaction of the excitatory inputs with the postsynaptic voltage-dependent ion channels will likely dominate the input-output relationship. the large SBCs described in cat (Osen, 1969) that projects to MSO bilaterally, rather than the smaller type of SBC reported in cat by Cant and Morest (1984). Note that the distinction between small and large SBCs has not been clearly shown in gerbil (Gleich et al., 1998; Bazwinksy et al., 2008).

\section{Juxtacellular recordings of spontaneous activity: interpretation of the waveforms}

The juxtacellular recording technique yielded a very good signal-to-noise ratio (Fig. $1 b-d$ ), allowing not only excellent isolation of single units but also a routine identification of successful and failed events. These could be distinguished by the presence or absence of a steep downward-deflecting phase (Fig. 1d). The complex events consisted of two or three phases. Based on previous studies (Pfeiffer, 1966; Guinan and Li, 1990; Lorteije et al., 2009; Typlt et al., 2010), we interpret them as follows. A small prespike (Fig. $1 d$, vertical arrows) is followed, after a brief delay, by a steeply positive-going phase (eEPSP) (Fig. $1 d$, tilted arrows). If EPSPs are sufficiently large, they trigger an AP. The supposed origin of the components and configuration leading to their recording is illustrated in Figure 1e, with prespike, eEPSP, and eAP labeled 1-3, respectively. The prespike is thought to result from the AP of a nearby endbulb of Held. The eAP reflects the somatic AP, which presumably has backpropagated after being triggered in the initial segment by a suprathreshold EPSP.

The possibility to also detect postsynaptic failures thus allows an analysis of the input-output relationship of single units in vivo. In addition, the good signal-to-noise ratio of the recordings allowed us to quantify the strength of individual synaptic events and the timing between eEPSPs and eAPs.

\section{Effects of EPSP size on failure}

eEPSP peak amplitude was strongly correlated with the maximum rising slope of the eEPSP (supplemental Fig. 1, available at www.jneurosci.org as supplemental material). Because rising slope is relatively insensitive to slow baseline fluctuations and to summation with APs (because APs are triggered with some delay), we quantify eEPSP size by its maximum rising slope, denoted eEPSP ${ }_{\text {max }}^{\prime}$, throughout this study. eEPSP size was a strong predictor of the success of individual events. This is illustrated in Figure $2 a$. A total of 1595 spontaneous events from one unit were time aligned at the steepest rising part of the eEPSP and divided into four categories according to eEPSP max $^{\prime}(<8,8-12,12-15$, and $>15 \mathrm{~V} / \mathrm{s}$ ). With increasing eEPSP size, the median waveforms of each category showed a gradual transition from failed events (Fig. $2 a$, lightest grayscale) via events evoking a delayed eAP to events evoking an eAP immediately following the rising flank of the eEPSP.

The median prespikes of each of the categories (Fig. $2 a$, inset) reveals that prespike size was not correlated with eEPSP size or transmission success. The delay between prespikes and eEPSPs was also similar across the categories. We only rarely observed systematic variations of prespike waveform with eEPSP size. These exceptions, however, lacked a monotonic correlation between prespike amplitude and eEPSP amplitude or slope. Together, these findings suggest that failed events originated from the same input source as successful events. Additional support for this conclusion is presented in supplemental Figure 2 (available at www.jneurosci.org as supplemental material).

The variation of eEPSP-eAP delay with eEPSP size, which is apparent from the median waveforms in Figure $2 a$, is plotted in Figure $2 b$ for the individual successful events from the same recording. Insets in Figure $2 b$ show two individual events. Apart 
a

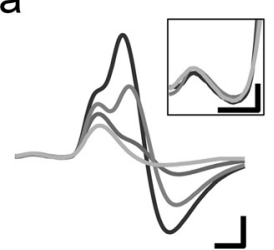

C

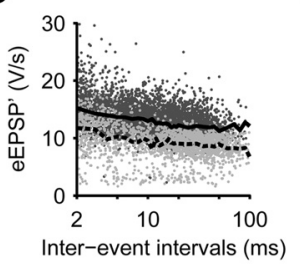

e

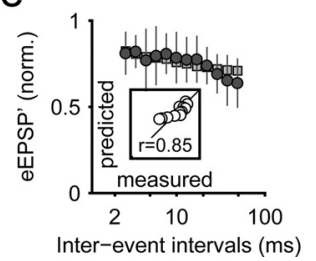

Figure 2. Maximum eEPSP slope and its correlates. $\boldsymbol{a}$, Median event waveforms categorized by the maximum steepness of the eEPSP component, eEPSP max $^{\prime}$. Shown is the same recording as in Figure $1 b-d$. Calibration: $1 \mathrm{mV}, 0.25 \mathrm{~ms}$. Inset, Magnification of prespike component for the four categories. Calibration: $0.1 \mathrm{mV}, 0.25 \mathrm{~ms}$. $\boldsymbol{b}$, Scatter plot of eEPSP- eAP delay versus eEPSP ${ }_{\max }^{\prime}$. Waveform insets illustrate timing of eEPSP ${ }_{\text {max }}^{\prime}(+)$ and eEPSP ${ }_{\text {max }}(\times)$. (RG09171u7) c, Scatter plot of eEPSP' ${ }_{\max }$ versus interevent interval. Dark and light symbols represent successful events and failures, respectively; solid and dashed lines indicate the corresponding binned averages. (RG09152u2) $\boldsymbol{d}$, Population data of normalized eEPSP ${ }_{\max }^{\prime}$ versus interevent interval. Lines represent binned averages; shaded areas indicate corresponding SD. $\boldsymbol{e}$, Binned averages of predicted eEPSP' ${ }_{\max }$ (squares) from an adaptive model (see Materials and Methods) fitted to the data of $c$ (pooling failed and successful events). Binned averaged data (circles) were normalized. Fitted model parameters are as follows: $11 \%$ facilitation per event; $\tau=11.1 \mathrm{~ms}$. Inset, Scatter plot of predictions and data, with correlation $r$ indicated.

from an overall shortening of eEPSP-eAP delay with eEPSP size, this analysis revealed that the smallest eEPSPs led to the largest spread of delays. Such variability is expected for EPSPs that just exceed the AP threshold and is consistent with in vivo recordings of the endbulb of Held synapse (Xu-Friedman and Regehr, 2005a; Yang and Xu-Friedman, 2009) and other systems (Rodriguez-Molina et al., 2007).

\section{Effects of interevent interval}

Figure $2 c$ shows eEPSP size of individual events as a function of the interevent interval [i.e., time elapsed since the previous event (whether a successful event or a failure)]. Dark and light symbols indicate successful and failed events, respectively. Trendlines were computed separately for successful and failed events. Shorter interevent intervals led to a larger eEPSP size, both for successful and failed events. This finding indicates the presence of short-term facilitation, rather than short-term depression, of synaptic events. Other factors such as summation of currents, which would cause a similar increase in synaptic strength at smaller intervals, cannot be ruled out. The facilitation of events at short intervals is a common finding in the units we analyzed, as shown in the population plot (Fig. $2 d$ ). We fitted a cumulative facilitation model (Varela et al., 1997) of the eEPSP' to episodes of spontaneous events from 38 SBCs and accepted fit parameters when correlation between predicted and measured eEPSP' was a

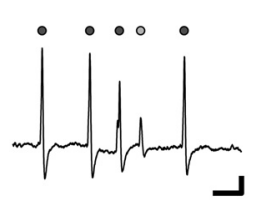

C

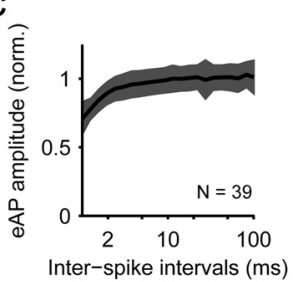

e

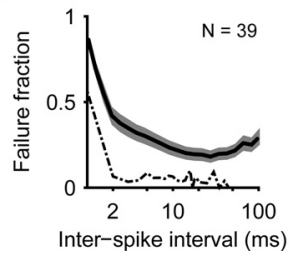

b

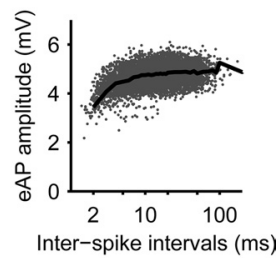

d

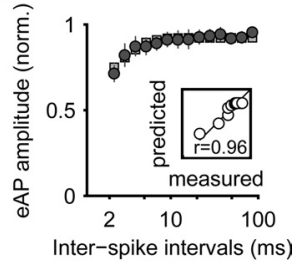

f

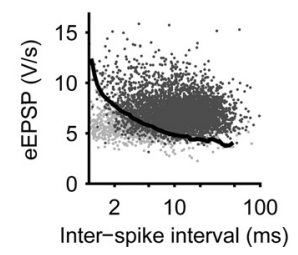

Figure 3. Spike depression and failures. $\boldsymbol{a}$, Spontaneous events illustrating progressive spike depression (dark gray circles) and failure (light gray circle). (RG09147u2) b, Scatter plot of eAP amplitude versus interspike interval. The solid line indicates binned average of eAP amplitude. (RG09174u4) c, Population data of normalized eAP amplitude versus interspike interval. Solid line, Population average; shaded area, SD. $\boldsymbol{d}$, Binned averages of predicted eAP amplitude (squares) from an adaptive spike-depression model (see Materials and Methods) fitted to the data. Binned averaged data (circles) were replotted from $\boldsymbol{b}$ after normalization. Fitted model parameters are as follows: fast depression factor, 0.31 per AP; $\tau_{\text {fast }}=1.5 \mathrm{~ms}$; slow depression factor, 0.9998 per AP; $\tau_{\text {slow }}=4.1$ s. Inset, Scatter plot comparing predicted and measured eAP amplitudes, with correlation $r$ indicated. (RG09171u4) $\boldsymbol{e}$, Failure fraction against interspike interval. Solid line, Population average; shaded area, SEM; dashed line, data for example unit RG09181u3.f, Sliding-window estimation of the optimal division (solid line) between successful events and failures (see text). (RG09181u3).

positive and significant ( $r>0 ; p<0.05$; see Materials and Methods). Decay of facilitation could be described by an exponential function with a time constant of $10.4 \pm 5.3 \mathrm{~ms}(n=12)$ with an average cumulative increase of $9 \pm 5 \%(n=12)$ eEPSP $^{\prime}$ per event. For an example, see Figure $2 e$. Average correlation coefficients between measured and predicted binned average eEPSP' were $r=0.66 \pm 0.14(n=12)$. Two of 38 units instead showed reduction $(-45$ and $-97 \%)$ of eEPSP' at very short intervals (time constants 2.74 and $1.99 \mathrm{~ms}$ ). Possibly, this reflected the refractory period of one of the smaller inputs.

The residual, noninterval-dependent variation in eEPSP size (Fig. 2c,d) may be explained, to a large extent, by the probabilistic nature of transmitter release. The good correlation between the size of the eEPSP and the presence or absence of failures suggests that the stochastic variability in the size of the eEPSPs played a large role in spike failures during spontaneous activity.

\section{Effects of interspike interval}

Another factor that played an important role in generating failures was postsynaptic spike depression. Trains of spontaneous eAPs separated by short intervals displayed a gradual reduction in amplitude; when accumulating, this depression often led to failures at very short interspike intervals (Fig. $3 a$ ). Full recovery from spike depression took several tens of milliseconds. To analyze the recovery, we plotted eAP amplitudes of spontaneous events 
against the interspike interval (i.e., time elapsed since the previous eAP). This revealed a clear depression of eAP amplitude at short intervals both in individual units (Fig. $3 b$ ) and in the population averages (Fig. 3c). We fitted a cumulative recovery model (Varela et al., 1997; Lorteije et al., 2009) of the eAP amplitude to episodes of spontaneous events from 38 SBCs and accepted fit parameters when correlation between predicted and measured eAP was positive and significant $(r>0 ; p<0.05$; see Materials and Methods). Recovery was described by the sum of two exponential functions with a fast time constant of $2.1 \pm 1.2 \mathrm{~ms}(n=$ 27). The slow component had relatively little impact and our data did not allow a closer analysis of this component. The recovery model was suited to predict the average interval-dependent change of eAP amplitudes (Fig. $3 d$ ), as signified by the good correlation $r=0.89 \pm 0.07(n=27)$ between average timebinned measured and predicted eAP amplitudes.

Failure rate increased sharply at short $(<5 \mathrm{~ms})$ interspike intervals (Fig. 3e). Although some individual units had very low failure rates at almost all intervals, very short $(<2 \mathrm{~ms})$ intervals caused higher failure rates in spontaneous events in almost all units. Despite the presence of synaptic facilitation (Fig. 2), average failure rates were $89 \pm 17 \%$ at an interval of $1 \mathrm{~ms}$, whereas they were only $21 \pm 24 \%$ at an interval of $10 \mathrm{~ms}$. Together, these results support the hypothesis that the relative refractory period of the postsynaptic membrane reduces the excitability of the SBC in an interspike interval-dependent manner. In some units, the failure rate increased again at very long $(>50 \mathrm{~ms})$ intervals (Fig. $3 e$ ). This may have been caused by the decay of short-term facilitation, or by a lack of long-range summation of postsynaptic events. Since interevent intervals $>50 \mathrm{~ms}$ occurred only rarely in our units, we did not pursue this phenomenon.

A consequence of the reduced excitability of the postsynaptic membrane is that larger EPSPs are needed to trigger spikes. APs transiently raise the threshold for subsequent EPSPs to evoke an AP. This important aspect of reduced excitability is not captured in the analysis of failure rate (Fig. 3e). To illustrate it, Figure $3 f$ shows eEPSP size of individual events as a function of the post-AP interval (time elapsed since the last eAP). The solid line indicates the optimal demarcation line separating failed events (Fig. $3 f$, light gray symbols) from successful events (Fig. $3 f$, black symbols) (see Materials and Methods for the computation of the threshold line). Although the populations of failed and successful events show some overlap, Figure $3 f$ indicates that the success of a given event (i.e., the ability of the eEPSP to trigger an eAP) is well predicted by a combination of two factors: (1) eEPSP size; and (2) interval since the previous eAP. This method of quantifying the threshold EPSP while taking post-AP time into account is used in the remainder of this study to isolate the effects of auditory stimulation on the excitability of SBCs.

\section{Increased failure rates during auditory stimulation}

In response to pure tones, SBCs showed an increased failure rate (Fig. $4 a, b$ ). In the example trace in Figure $4 a$, a $1500 \mathrm{~Hz}$ tone presented at $30 \mathrm{~dB}$ SPL evoked an input rate of 356 events/s and an output rate of $228 \mathrm{spikes/s} \mathrm{(failure} \mathrm{rate,} 36 \%$ ). When increasing the intensity to $60 \mathrm{~dB} \mathrm{SPL}$ (Fig. $4 b$ ), the input and output rates became 420 events/s and 148 spikes/s, respectively (failure rate, $65 \%$ ). Similar findings were previously reported by Englitz et al. (2009). High failure rates were typically accompanied by a strong reduction in eAP amplitude in the sustained part of the response, even at interspike intervals $>10 \mathrm{~ms}$ (Fig. $4 b$ ).

Figure $4 c$ compares the monotonic input rate (squares) and the nonmonotonic output rate (circles). Transmission was essen-
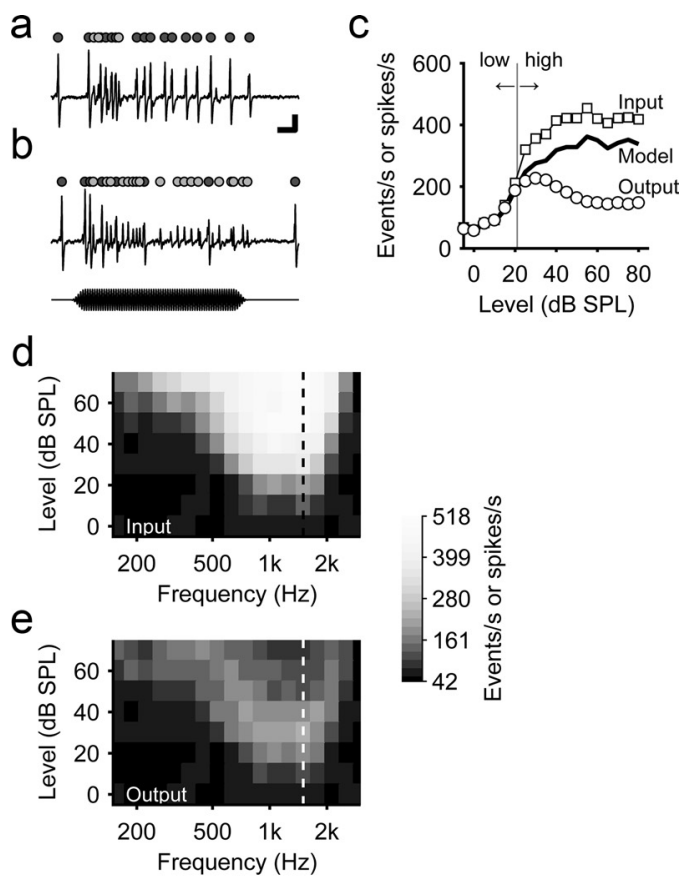

$f$

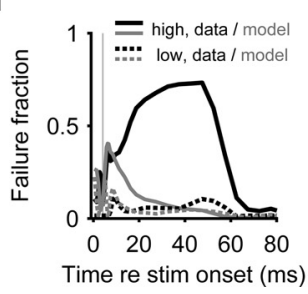

9

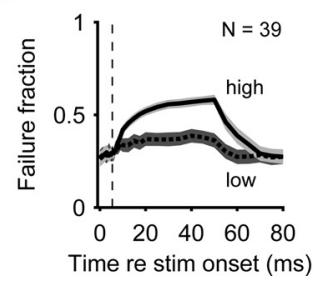

Figure 4. Increased failure rate during stimulation. $\boldsymbol{a}$, Response to a $50 \mathrm{~ms}, 1500 \mathrm{~Hz}$ tone at $30 \mathrm{~dB}$ SPL. Calibration: $3 \mathrm{mV}, 5 \mathrm{~ms}$. (RG09181u3) $\boldsymbol{b}$, Response of the same unit to the same tone, now at $60 \mathrm{~dB}$ SPL. Notice the increased incidence of failures (light gray circles). Stimulus waveform is shown below the recording. c, Rate-level curves for eEPSP rate ( $\square$, Input), AP rate $(\bigcirc$ Output), and predicted output rate (solid line, Model) based on interval dependency alone (see Fig. $3 e, f)$. The vertical line and arrows denote the border between stimulus levels causing low and high failure rates. The dashed line indicates spontaneous AP rate. (RG09181u3) $\boldsymbol{d}, \boldsymbol{e}$, Frequencyresponse areas of all events $(\boldsymbol{d})$, representing the total input to the cell, and of eAPs $(\boldsymbol{e})$, representing its output. Rates are represented by grayscales as indicated next to the graph. Vertical lines mark the conditions of the rate-level curve of c.f. Failure fraction (see Fig. $3 e$ ) against time after stimulus onset. Thick black lines represent measured data from low SPL (dashed) and high SPL (solid) conditions. Thin gray lines are predictions based on interval dependency alone ( $\boldsymbol{c}$; dashed, low SPL; solid, high SPL). The light gray vertical line marks first-spike latency. (RG09181u3) g, Population means (lines) and SEM (shaded area) of failure fraction against time after stimulus onset for 39 units. Top curve, High SPL conditions ( $51 \pm 11 \mathrm{~dB}$ above threshold) bottom curve, low SPL (18 $\pm 6 \mathrm{~dB}$ above threshold) conditions. The vertical line indicates average first-spike latency for the same 39 units.

tially faithful up to $20 \mathrm{~dB}$ SPL; at higher intensities, input and output rates strongly diverged. Importantly, the growth of failure rate cannot be accounted for by the interval-dependent spike depression analyzed earlier (Fig. 3, compare $c, d$ ). The solid line (no symbols) in Figure $4 c$ shows the spike rate predicted by a first-order spike-depression model derived from the spontaneous activity of the same unit and applied to its driven input events (see Materials and Methods). The model predicts a moderate, saturating output rate (as expected from the self-limiting character of spike depression). It greatly overestimates the measured output rate and completely misses its nonmonotonicity. We conclude that an additional mechanism, different from the mechanism underlying failures in spontaneous events, acted on the SBC during sound stimulation. 
The increased failure rates had a strong impact on the response of the neuron. Whereas the tuning of inputs [frequencyresponse area (FRA)] (Fig. 4d) was primary like, the tuning of the outputs (Fig. 4e) was complex and nonmonotonic, although CF and threshold did not differ from the tuning of the inputs. Steep growth of the failure rate resulting in nonmonotonic responses and complex response areas was observed in 24 of the 39 SBCs.

The poststimulus time course of the failure rate is shown in Figure $4 f$ separately for low ( $\leq 20 \mathrm{~dB}$ SPL) and high ( $>20 \mathrm{~dB}$ SPL) stimulus levels. This division was based on the divergence of the rate-level curves in Figure $4 c$. The vertical line in Figure $4 f$ marks the first-spike latency ("response onset"). The failure rate for the lower SPLs (Fig. 4f, black dashed line) showed a small but steep rise starting $\sim 2 \mathrm{~ms}$ after response onset, which is fairly well predicted by the first-order spike-depression model (Fig. $4 f$, gray dashed line). In contrast, the high SPL failure rate (Fig. $4 f$, black solid line) showed a two-stage progression. A steep initial rise, 1-2 ms after response onset, was followed by a slower rise starting $\sim 5 \mathrm{~ms}$ after response onset. The spike-depression model (Fig. $4 f$, gray solid line) predicts the initial rise but completely misses the second, slower increase in the failure rate. The slowly saturating growth of the failure rate was a common finding in the responses to higher SPL tones (Fig. $4 g$, population plot).

We tested the potential contribution of changes in the strength of excitatory transmission to the observed increased failure rates by comparing the distributions of eEPSP size and prespike amplitude between low and high SPL conditions. In the unit shown (supplemental Fig. 3. available at www.jneurosci.org as supplemental material), failure rates were 6 and $65 \%$ for the low and high SPL condition, respectively. Surprisingly, neither eEPSP size nor prespike amplitude differed systematically between the two conditions. In other units, the distribution of eEPSP sizes shifted toward slightly higher values with increasing SPL. In some of those units, the larger eEPSPs were also preceded by larger prespikes. These observations are consistent with the existence of multiple, converging inputs onto SBCs, the secondary inputs only being recruited at sufficient SPL. Our dataset, however, did not allow a systematic analysis of these effects. Importantly, we did not find any indication for a reduced strength of synaptic transmission underlying the increased failure during auditory stimulation. This points at a postsynaptic origin of the increased failure rates, and the recruitment of inhibitory inputs is a likely candidate (Englitz et al., 2009).

\section{Sound-evoked elevation of threshold EPSP}

We next determined the optimal boundary between failed and successful events in scatter plots of eEPSP size versus post-eAP interval (see Fig. $3 f$ for spontaneous events). In Figure $5 a$, the resulting threshold EPSP values are plotted separately for low SPL conditions (dashed line) and high SPL conditions (solid line). For short ( $<2 \mathrm{~ms}$ ) intervals, spike depression dominated, causing the two curves to converge. For longer intervals, a systematic difference between the curves was observed: threshold EPSP was elevated for high SPL conditions. This sound-induced threshold elevation was primarily independent of interval. The threshold elevation is also illustrated in Figure 5, $b$ and $c$, where the median waveforms were computed for given ranges of eEPSP size (see Fig. $2 a$ for spontaneous events). The same eEPSP sizes that readily evoked eAPs in low SPL conditions (Fig. $5 c$ ) failed to do so in high SPL conditions (Fig. 5b). These observations underline the fact that the sound-evoked increase in failure rate is not caused by a reduced eEPSP size but by a systematic increase in the threshold
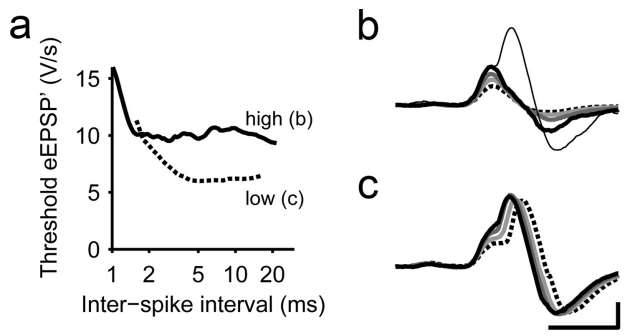

d

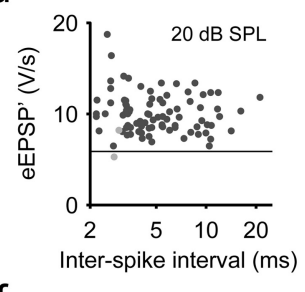

e
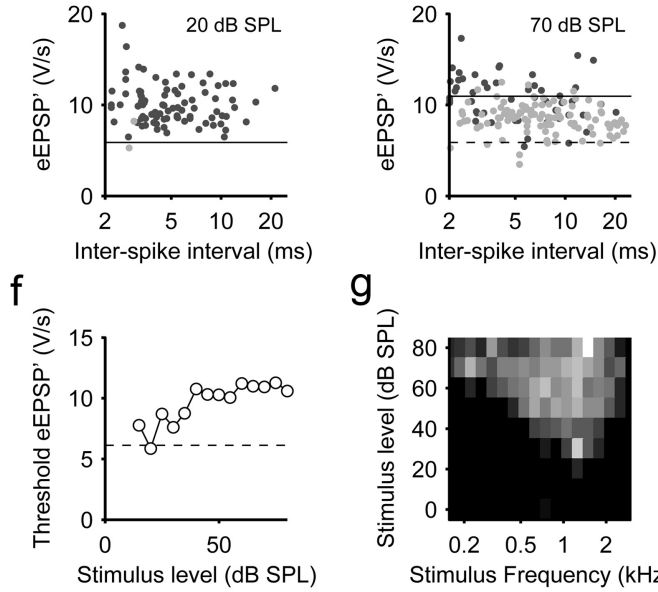

g

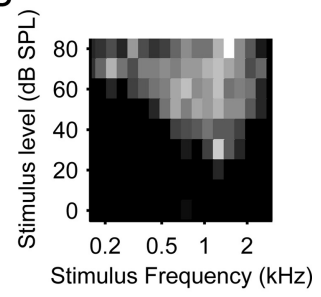

$\mathrm{h}$

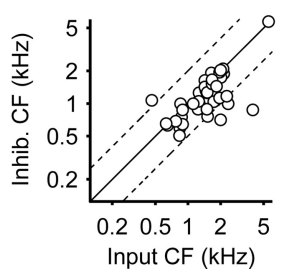

i

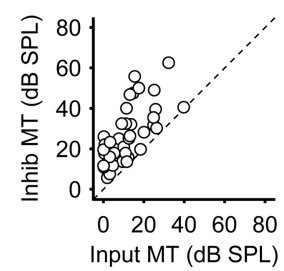

Figure 5. Elevation of threshold EPSP during stimulation. $\boldsymbol{a}$, Threshold eEPSP' max $_{\text {ax }}$ (strength of eEPSP needed to just evoke an AP) against interspike interval for a lowSPL (dashed line) and a high SPL (solid line) condition. $\boldsymbol{b}, \boldsymbol{c}$, Mean waveforms of events categorized by their eEPSP' ${ }_{\max }$. Category boundaries were $7.5,10$, and $12.5 \mathrm{~V} / \mathrm{s}$. The same categories were used for low SPLS (c) and high SPLS $(\boldsymbol{b})$, revealing that at high SPLs, larger EPSPs are needed to trigger an AP. $\boldsymbol{d}, \boldsymbol{e}$, eEPSP ${ }_{\max }^{\prime}$ versus interspike interval in the interval-independent range for a $20 \mathrm{~dB} \mathrm{SPL}$ (d) and $70 \mathrm{~dB}$ SPL $(\boldsymbol{e})$ condition. The solid line demarcates threshold eEPSP' ${ }_{\max }$ [i.e., the optimal division between failures (light gray) and successful events (dark gray)]. The threshold line of $\boldsymbol{d}$ is replotted in $\boldsymbol{e}$ (dashed line) for comparison. Events for intervals $<2$ ms are omitted. $\boldsymbol{f}$, Threshold eEPSP max $^{\prime}$ against SPL of a $1500 \mathrm{~Hz}$ tone. The dashed line indicates threshold from spontaneous recordings. Lack of failures prevented threshold estimation for $0-10$ dB SPL. $g$, Frequency-response area of threshold elevation. Threshold values are represented by grayscale. Tuning of threshold elevation was similar to tuning of event rate and AP rate of the same unit (see Fig. $4 d, e) .(\boldsymbol{a}-\boldsymbol{g}, \mathrm{RG09181u3)} \boldsymbol{h}$, Comparison of excitatory CFs (from AP response areas) and inhibitory CFs (from threshold elevation areas). Solid lines indicate equality and \pm 1 octave. Data are from 35 units. $i$, Comparison of excitatory and inhibitory minimal thresholds (MT) for the same data as in $\boldsymbol{h}$. Inhib., inhibitory.

EPSP, the minimum size of the eEPSP reliably associated with an $\mathrm{AP}$, in the postsynaptic cell.

From the threshold EPSP curves (Fig. 5a), we chose a lower limit of post-eAP interval beyond which the sound-evoked threshold elevation was primarily independent of interval. By pooling all the events having intervals above this lower limit, we were able to determine the threshold eEPSP for individual stimulus conditions, without the need for pooling across SPLs. This procedure is illustrated for a $1500 \mathrm{~Hz}$ tone presented at $20 \mathrm{~dB}$ SPL (Fig. $5 d$ ) and $70 \mathrm{~dB}$ SPL (Fig. 5e). The resulting threshold EPSP versus SPL curve for $1500 \mathrm{~Hz}$ tones is shown in Figure $5 f$. 
We propose to use the elevation of the threshold EPSP as a metric for inhibition. Further evidence that synaptic inhibition can cause the observed changes in the threshold EPSP comes from a simulation that will be presented below. This metric is primarily independent of input and output rates and their ratio. In this interpretation, the curve in Figure $5 f$ reflects the monotonic growth of inhibition with SPL, saturating at the highest SPLs.

Our method of quantifying inhibition allows the mapping of inhibitory receptive fields from single-tone responses. The resulting inhibitory FRA is shown in Figure $5 g$. Importantly, tuning of inhibition was primary like and similar to the tuning of excitation (shown in Fig. $4, d$ and $e$, for the same unit), with similar CF but higher threshold. The contiguous character of the inhibitory FRA, continuously extending into the low-frequency tail, dispenses with the need for postulating a separate, below CF inhibitory input to describe the low-frequency inhibitory "sideband" routinely found in two-tone data [AN (Schmiedt, 1982) and AVCN (Kopp-Scheinpflug et al., 2002)].

When comparing the inhibitory FRA (Fig. $5 g$ ) to the two excitatory FRAs (Fig. 4), the inhibitory FRA has exactly the shape that is needed to turn the tuning of the input (Fig. $4 d$ ) into the tuning of the output (Fig. 4e). Over a wide range of stimulus frequencies, inhibitory thresholds were 10-20 dB higher than excitatory thresholds, resulting in a near equality of rate thresholds for input and output. The combination of this threshold difference with the steep growth of inhibition above threshold (Fig. 5f) accounts for the band-like character of the excitatory output FRA (Fig. 4e).

We successfully constructed maps of the tuning of inhibition for 35 of 39 SBCs. Almost all (34 of 35, 97\%) of the maps showed continuous areas of inhibition. The CFs of inhibition and excitation deviated, on average, by $-0.3 \pm 0.6$ octaves (Fig. $5 g$ ). In the few cases in which the CFs differed markedly, inhibition was tuned to lower frequencies (five of six, 83\%). The threshold of inhibition was higher than the threshold of excitation (average, $15 \pm 11 \mathrm{~dB}$ ) (Fig. 5i). In some instances, the area of inhibition extended to slightly higher frequencies than the excitation (19 of $35,54 \%)$ at high-stimulus intensities. In these cases, the upper flank of the inhibitory FRA was less steep than the upper flank of the excitatory FRA. On average, however, we found no differences in $\mathrm{Q}_{10}$ values between inhibition and excitation. In 45\% (16 of 35) of the units, the inhibitory area was narrower than the excitatory area. This was mostly (13 of $16,81 \%)$ caused by an absence of inhibition at low frequencies.

In brief, the large overlap between excitatory and inhibitory receptive fields was a common finding in our data, suggesting a dominance of same CF inhibitory inputs (Wickesberg and Oertel, 1988, 1990) and only little impact of widely tuned inhibitory sources (Ferragamo et al., 1998).

\section{Modeling the interaction of excitatory and inhibitory inputs to SBCs}

Our data indicate that inhibitory input to the SBC raises the threshold EPSP, reduces eAP amplitude (Fig. $4 b$ ), but hardly affects eEPSP size. What type of inhibitory input can produce this combination of effects? First of all, modulatory (e.g., monoaminergic) inputs, which have been described to be present in the AVCN (Klepper and Herbert, 1991), can essentially be excluded. The precise tuning (Fig. 5h) and the relatively rapid onset of the effect (Fig. $4 f$ ) argue against diffuse, modulatory transmission and in favor of a more direct inhibitory input. Any hyperpolarizing IPSP will have two concurrent effects on the postsynaptic membrane. First, the hyperpolarization pulls the membrane potential away from the AP threshold, causing excitatory inputs that would be suprathreshold at rest to fail. Second, the temporary increase in membrane conductance shunts EPSCs. Whereas in the avian auditory brainstem shunting dominates (Howard et al., 2007), it is generally agreed that inhibition in SBCs of the mammalian cochlear nucleus stems from glycinergic (Wu and Oertel, 1986; Kopp-Scheinpflug et al., 2002; Milenkovic et al., 2007) and GABAergic (Caspary et al., 1994) synapses eliciting hyperpolarizing IPSPs (Wickesberg and Oertel, 1990; Paolini and Clark, 1998).

We modeled the interaction of strong and fast excitatory inputs and hyperpolarizing synaptic inhibition. An established model of SBCs (Rothman and Manis, 2003) was extended to a compartmental model consisting of a soma, a spike initiation site, and a stretch of axon. The synaptic inputs contacted the soma and were spatially separated. The voltage-gated sodium conductance was confined to the initiation site. Repeating all simulations using an active soma produced only quantitatively, but not qualitatively, different results. Furthermore, the outcome of the simulations was primarily insensitive to changing the strengths of low- and highvoltage-activated potassium conductance and of $I_{\mathrm{h}}$ to the ranges occurring in small SBCs, as reported by Cao et al. (2007). In contrast, eAP depression was influenced by the amount of lowvoltage activated potassium conductance, suggesting that potassium channel activation can contribute to spike depression. We did not include the bushy dendrites, whose role in the SBC physiology is primarily unknown. See Materials and Methods for more details of the model. Our simulations indicate that, although potassium channel activation (supplemental Figs. 4 and 5, available at www.jneurosci.org as supplemental material) and sodium channel inactivation (supplemental Fig. 6, available at www.jneurosci.org as supplemental material) may contribute to changes in threshold EPSPs, it is unlikely that they are responsible for the stimulus-driven, frequency-tuned rise in threshold EPSP that we observed, especially considering its general independence of spike rate.

We next used the simulation to test how known inhibitory mechanisms affect the threshold EPSP. We first tested how a pure hyperpolarization, with a negligible increase in membrane conductance, changed the effect of excitatory inputs to the model. The simulations (Fig. 6a1-a3) revealed that, with an increasing hyperpolarizing current, the amplitudes of both the intracellular EPSP (iEPSP) (Fig. 6a1) and its time derivative (iEPSP') (Fig. $6 a 2$ ), which served as an approximation of the extracellularly recorded potentials (Lorteije et al., 2009), strongly increased because of the greater driving force of the excitatory postsynaptic conductance (the current was simulated to reverse at $0 \mathrm{mV}$ ). Maximum iEPSP" value, the analog of eEPSP $_{\text {max }}^{\prime}$ used in this study to quantify EPSP size (Figs. 2, 3, 5), grew steeply with a hyperpolarizing current (Fig. 6a3, solid line). The threshold EPSP, defined as the minimal iEPSP" that elicited an AP in the model neuron, also increased steeply (Fig. 6a3, dashed line).

We next tested how a pure shunting-type inhibition, simulated by a continuous increase in leak conductance, interacted with the excitatory input (Fig. 6b). By design, the increased leak conductance did not change the membrane potential, but it did produce marked reductions in iEPSP' (Fig. 6b1) and iEPSP" (Fig. 6b2) amplitude. With increasing shunting conductance, peak iEPSP" was slightly reduced, and the simulated threshold EPSP increased steeply (Fig. 6b3).

We conclude that neither a purely hyperpolarizing inhibition type nor a pure shunting inhibition type is consistent with our 
juxtacellular recordings (compare Fig. 6, $a 2$ and $b 2$, with Fig. 5, $b$ and $c$ ). The contrasting ways in which the two models failed to reproduce the data suggested that a combined effect of hyperpolarization and shunting might be more successful. We therefore implemented an inhibitory input defined by a conductancebased synapse with a reversal potential more negative than the resting potential. We first explored the timing and amount of conductance needed to affect the excitatory input and found that the inhibitory event(s) must lead the excitatory event and the strength of the inhibitory conductance must match the excitatory conductance. The resulting iEPSP and iEPSP' were hardly affected by the interaction with the inhibitory input (Fig. $6 c 1, c 2$ ), because the opposing effects of hyperpolarization and shunting effectively canceled each other. With increasing inhibitory synaptic conductance, peak iEPSP" increased slightly (Fig. 6c3, solid line), whereas the threshold EPSP increased more steeply (Fig. 6c3, dashed line).

Thus, a model based on an appropriate combination of hyperpolarization and shunting-type inhibition correctly predicts the elevated threshold EPSP and near-constant EPSP size observed in our juxtacellular recordings. Our data thus indicate that an increase in the strength of excitatory synaptic transmission is not needed to reproduce the observed effects on the extracellular waveforms, but we cannot exclude that an underlying increase in excitatory synaptic transmission is compensated by a stronger shuntingtype inhibition. The required amount of inhibitory conductance is unlikely to originate from a single IPSP just preceding the EPSP (Wickesberg and Oertel, 1988;

Wickesberg and Oertel, 1990; Paolini and Clark, 1998). We therefore simulated the effect of multiple, small IPSCs, leading to a slow buildup of inhibition (see Materials and Methods). When stimulated with a train of EPSCs (3 ms interval), the membrane potential was gradually hyperpolarized, because the IPSPs lasted longer than the EPSPs. The membrane potential and its time derivative are shown in Figure $6, d 1$ and $d 2$. These simulations reproduce the main aspects of our juxtacellular recordings: a gradual decline of AP size culminating in sustained failure; increase in EPSP-AP latency; and near constancy of EPSP size.

We conclude that the response characteristics of SBCs stimulated by moderate and high SPL tones are well captured by the interaction of strong excitatory synaptic inputs and weaker, temporally summating, inhibitory synaptic inputs. These characteristics are consistent with the action of hyperpolarizing glycinergic inhibitory synapses reported to terminate on SBCs of the AVCN (Wickesberg and Oertel, 1988; Paolini and Clark, 1998).

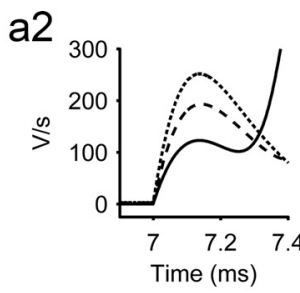

a3

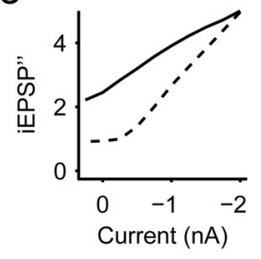

b3
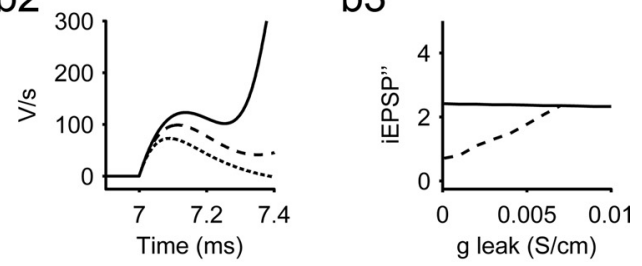

c3
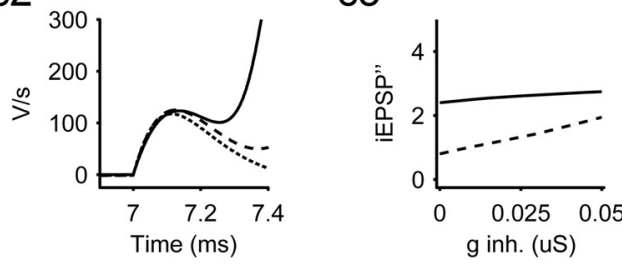

d2

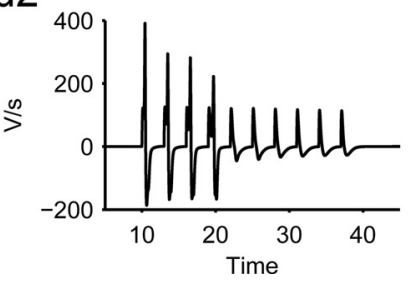

Figure 6. Modeling inhibition. The first derivative iEPSP' (plot 2 in rows a-c) of the simulated membrane potential iEPSP (plot derivative iEPSP". Simulated spike threshold is depicted by the dashed line. $\boldsymbol{a}$, Effect of interaction of a weak (solid line, $-0.25 \mathrm{nA}$ ), (mimicking shunting inhibition) with excitatory synaptic events (solid line, $0.001 \mathrm{~S} / \mathrm{cm}^{2}$; dashed line, $0.005 \mathrm{~S} / \mathrm{cm}^{2}$; (3 ms interval). Summation of inhibitory conductance leads to a gradual elevation of spike threshold similar to our findings in vivo (see Fig. 4b). Notice the strong reduction of AP amplitude whereas EPSP amplitudes remain unaffected.

\section{Phase locking}

So far, we have analyzed the input-output relationship of SBCs in terms of failure rate (i.e., the fraction of subthreshold eEPSPs). The selective transmission of SBCs, however, is also known to affect temporal precision (Dehmel et al., 2010), with obvious functional implications for binaural processing. We compared the timing of postsynaptic events (eEPSPs and eAPs) and analyzed the complex extracellular waveforms to identify the cellular mechanisms shaping the temporal precision of the SBC output.

The timing of individual events with respect to the stimulus phase is shown in Figure $7 a$ for a $30 \mathrm{~dB}$ SPL pure-tone stimulus. The eEPSPs (Fig. 7a, circles) occurred at a variety of phase positions, indicating nonperfect phase locking. Some, but not all, eEPSPs were followed by eAPs (Fig. $7 a$, squares). The phase locking of eAPs is affected by two factors: (1) the selection of eEPSPs that do or do not evoke an eAP; and (2) the variable delay between a successful eEPSP and the eAP it evokes. These two factors are clearly illustrated in the response to the $65 \mathrm{~dB}$ SPL tone (Fig. 7b), which shows a higher failure rate. The selection of successful 
a

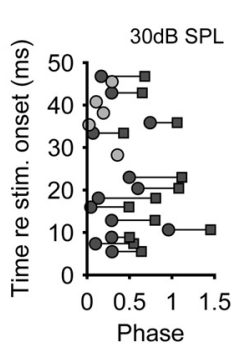

d

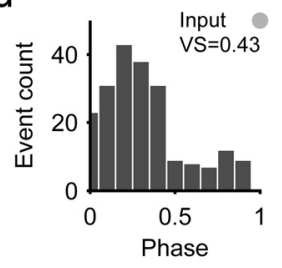

g

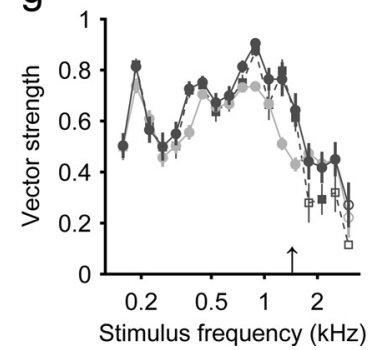

b

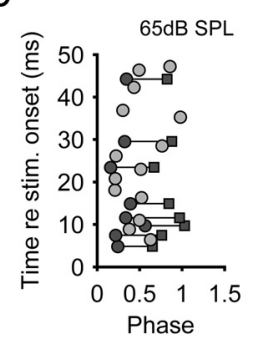

e

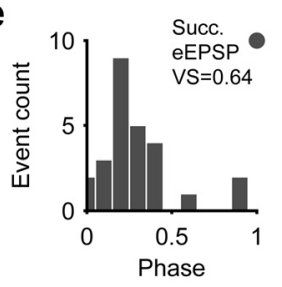

C

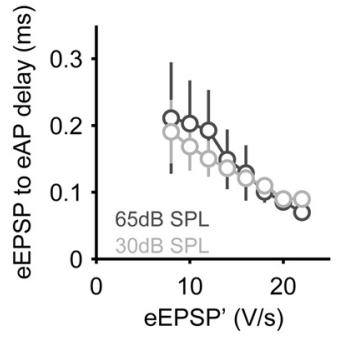

f

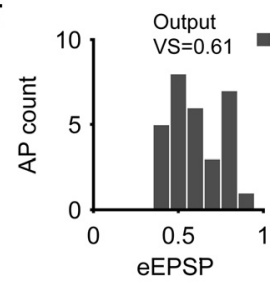

$\mathrm{h}$

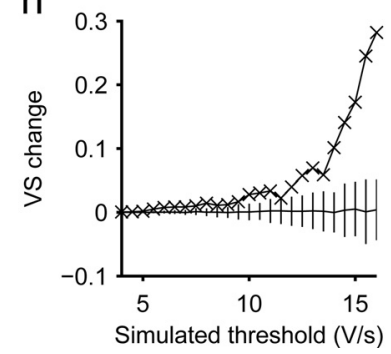

Figure 7. Effect of threshold elevation on phase locking. $\boldsymbol{a}, \boldsymbol{b}$, Timing of events relative to stimulus phase. Symbols represent the timing of failed (light gray) and successful (dark gray) eEPSPs and corresponding eAPs $(\boldsymbol{\square})$ for a $1500 \mathrm{~Hz}$ tone at $30 \mathrm{dBSPL}$ (a; weak threshold elevation) and $65 \mathrm{~dB} \mathrm{SPL} \mathrm{(b}$; strong threshold elevation). $\boldsymbol{c}$, Delay between eEPSP and eAP plotted against eEPSP' ${ }_{\max }$ for the two SPLs. $\boldsymbol{d}-\boldsymbol{f}$, Separate cycle histograms for input (all events), successful events only, and output (eAPs). eEPSPs were timed on their steepest rising slope, and eAPs were timed on their peak. The stimulus was $1500 \mathrm{~Hz}, 70 \mathrm{~dB}$ SPL. Vector strength VS is indicated in the graphs. $\boldsymbol{g}$, Variation of VS with frequency of $70 \mathrm{~dB}$ SPL tones, separately for all eEPSPs, successful eEPSPs only, and eAPs (symbols as in panels $\boldsymbol{d}-\boldsymbol{f}$; filled symbols signify Rayleigh $p<0.001$ ). Error bars are bootstrapped variability estimates (see Materials and Methods). The arrow marks (F. $\boldsymbol{h}$, Simulation of improvement in phase locking achieved by selection of large events. Top curve, Increase in VS between input events and selected events, plotted against imposed threshold. The stimulus was $1500 \mathrm{~Hz}$, $0-80 \mathrm{~dB}$ SPL. Bottom curve, effect on VS caused of random selection of events. Error bars are as in $\boldsymbol{g}$. (all panels: RG09181u3).

eEPSPs (the first factor) appeared to favor a subset of events that was confined to a narrower range of phases than the set of all input events. Such a preference for well-timed inputs may well induce an actual improvement of phase locking. In contrast, the variability in eEPSP-eAP delay (the second factor) can only result in loss of phase locking.

We tested whether the sound-induced elevation of threshold EPSP affected the relative timing of eEPSPs and eAPs and, more specifically, the variability in their relative delay. Figure $7 c$ shows average eEPSP-eAP delay binned according to eEPSP size, in which spontaneous events are compared to driven events. For both conditions, variability and absolute delay were reduced with increasing eEPSP size, but overall variability was similar. Thus, the temporal jitter of eAP initiation was not affected by the elevation of threshold EPSP.

Cycle histograms of total input (all eEPSPs), successful input only, and output (eAPs) are presented in Figure $7 d-f$, for a $1500 \mathrm{~Hz}, 70 \mathrm{~dB}$ SPL tone. The phase distribution of successful eEPSPs (Fig. 7e) was narrower and sharper than that of the total input (Fig. $7 d$ ). The phase distribution of the output (Fig. $7 f$ ) was somewhat broader than the successful eEPSP distribution but still narrower than the input distribution. In this example, the sharpening effect of the eEPSP selection (Fig. 7, compare $d, e$ ) ex- ceeds the smearing effect of temporal jitter (Fig. 7, compare $e, f$ ), causing a net improvement of phase locking.

Figure $7 g$ shows vector strength as a function of the frequency of a $70 \mathrm{~dB}$ SPL tone. The three curves represent all eEPSPs, successful eEPSPs, and eAPs. The improvement in phase locking of successful eEPSPs versus all eEPSPs occurred only in a limited frequency range, in this case between 300 and $1500 \mathrm{~Hz}$. This was the same range that displayed a sizeable threshold EPSP elevation, as seen in the "inhibitory FRA" shown earlier for the same unit (Fig. 5g).

The improvement of phase locking, combined with our earlier observation that eEPSP size is a strong predictor of success for individual events (Fig. 2d), suggests that larger eEPSPs are better synchronized to the stimulus than smaller eEPSPs. To test this assumption, we took all inputs for a given stimulus condition (successful and failed events alike) and selected the subset of events whose size exceeded a given threshold. The increase in vector strength achieved by this thresholding procedure is plotted against the threshold criterion in Figure $7 \mathrm{~h}$. The thresholding yielded a substantial improvement in phase locking. To check that this was not attributable to the mere reduction of event counts, we randomly removed the same number of events as were rejected by the thresholding (Fig. $7 h$, bottom curve). This random rejection increased the variability (Fig. $7 h$, error bars) but not the mean value of vector strength. We conclude that, within the totality of synaptic events reaching the SBC, larger events were relatively better phase locked

to the stimulus.

Population data on phase locking are shown in Figure 8. We included all recordings obtained with tones between 500 and $1500 \mathrm{~Hz}$ (the most common range of inhibitory threshold elevation) that yielded significant phase locking $(p<0.001$, Rayleigh test). This resulted in a dataset consisting of 1307 individual stimulus conditions from 39 units. Further restriction to conditions for which the threshold EPSP could also be determined resulted in a dataset containing 617 individual stimulus conditions from 39 units.

The effect of selective success was evaluated by plotting vector strength of successful events against vector strength of all events (Fig. 8a). Most symbols lie above the line of equality. This indicates that, in the majority of recordings, successful events are better phase locked than the totality of input events. The effect of variable eEPSP-eAP delay is shown in a scatter plot comparing vector strengths between successful events and eAPs (Fig. 8b). As expected, this effect is generally detrimental. We evaluated the combined effect of the two transformations shown in Figure 8, $a$ and $b$, by using vector strength of all input events as a baseline reference in conditions for which the threshold EPSP was determined. The resulting distributions of vector strength difference are shown in Figure $8 c$. This analysis confirms the clear 
a

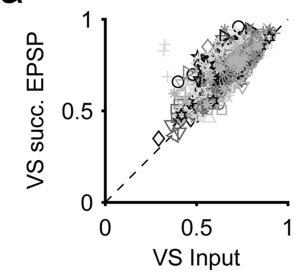

C

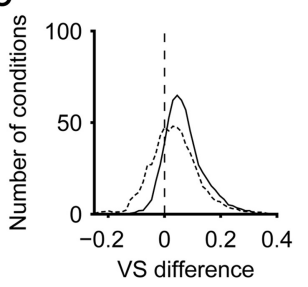

b

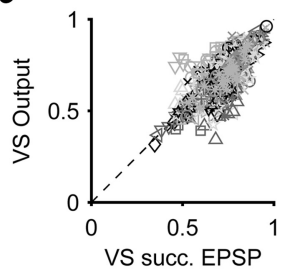

d

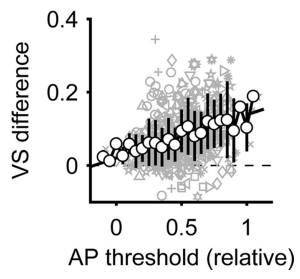

Figure 8. Transformation of phase locking by SBCs (population data). $\boldsymbol{a}$, Scatter plot of vector strength VS for all EPSPs (Input; $x$-axis) and successful events only ( $y$-axis). Each symbol represents a single stimulus condition (SPL, frequency); marker types signify different units. A total of 1307 conditions from 39 units is included, based on Rayleigh significance ( $p<0.001$ ) and stimulus frequency $(500-1500 \mathrm{~Hz})$. In $84 \%$ (1096 of 1307) of the cases, successful events were better synchronized than total input. The dashed line indicates line of identity. $\boldsymbol{b}$, Scatter plot of VS for successful events ( $x$-axis) and APs (Output; $y$-axis). Layout is as in $\boldsymbol{a}$. In 75\% (981 of 1307) of the cases, VS of APs is smaller than VS of successful events. c, Distributions of VS transformations. Solid line, Input versus successful events; dashed line, input versus output. The dataset is now restricted to 617 conditions from 39 units. $\boldsymbol{d}$, Difference in VS (input vs successful events) plotted against elevation of threshold eEPSP' ${ }_{\max }$ (see Fig. 7). Each light gray marker represents a VS value from a single stimulus condition. Marker types indicate different units; same dataset as in c. Open circles show binned means and SD. The solid black line shows linear regression $\left(y=0.11 x+0.03 ; r^{2}=0.805\right)$. The dashed line represents line of zero difference.

improvement of phase locking at the stage of successful eEPSPs (Fig. $8 c$, solid line) (92\% of points above the line of zero difference) and shows how this improvement is reduced, but not eliminated, at the stage of APs (Fig. $8 c$, dashed line) $(68 \%$ above zero-difference line). Both distributions significantly differed from zero mean (Kolmogorov-Smirnov test, $p<0.001$ ) and from each other (Kolmogorov-Smirnov test, $p<0.001$ ). Thus, although the jitter introduced by AP initiation degraded the temporal precision of the signal relayed by SBCs, this was positively offset by the gain in synchronization accomplished by the selection of synaptic events.

Our final analysis addresses the relationship between enhanced synchronization and threshold EPSP elevation (Fig. 8d) (see Materials and Methods for details), using the same set of 617 recordings as shown in Figure $8 c$. The modest, but systematic, increase in vector strength enhancement with threshold elevation suggests a functional role of inhibition in the enhancement (or preservation) of temporal precision of SBCs. In this scenario, the high input event rates evoked by intense sounds are accompanied by a hyperpolarizing inhibitory input, which raises the threshold EPSP. As a result, only the larger EPSPs, which are better phase locked to the stimulus, manage to evoke APs. At low sound intensities, when input rates are lower, the threshold EPSP is lowered. The upshot is a selective type of dynamic range compression, in which the event rate is traded for temporal precision.

\section{Discussion}

We dissected the intrinsic and network mechanisms controlling the input-output relationship of SBCs in vivo. Without auditory stimulation, postsynaptic spike depression and stochastic fluctuations in EPSP size were the main factors determining jitter and reliability of the endbulb synapse, whereas no evidence for a con-

tribution of short-term synaptic depression was found. During auditory stimulation, the recruitment of tuned synaptic inhibition caused postsynaptic spikes to be preferentially triggered by well-timed, large EPSPs, thus improving phase locking.

\section{Low release probability of the endbulb of Held in vivo}

In vivo, SBCs operated close to AP threshold. Most recordings showed postsynaptic failures during spontaneous activity, in agreement with previous work (Englitz et al., 2009). A major contributor to postsynaptic failure was spike depression, presumably dominated by sodium channel inactivation (Henze and Buzsáki, 2001; Azouz and Gray, 2000; Platkiewicz and Brette, 2010). At longer intervals, spike depression was minimal, but failures generally still occurred, associated with relatively small EPSPs. Since the prespike, on average, was uncorrelated with EPSP size, we hypothesize that small and large EPSPs share the same presynaptic origin and that their different sizes reflect stochastic fluctuations in synaptic strength. The presence of postsynaptic failures and absence of synaptic depression suggest that the release probability of the endbulb of Held is lower in vivo than in slices. Previously, no effect of interspike interval on synaptic strength was observed for bushy cells, although the endbulb synapse was not studied (Young and Sachs, 2008).

Our data contrast sharply with slice studies reporting that the endbulb synapse is suprathreshold by a large margin in the resting state and exhibits strong synaptic depression even under conditions designed to mimic the in vivo situation (Chanda and $\mathrm{Xu}$-Friedman, 2010a). Slice studies suggest that the release of maximally 20 vesicles suffices to trigger a spike in SBCs (Isaacson and Walmsley, 1996; Chanda and Xu-Friedman, 2010a). Around threshold, the average release probability per release site is $<0.03$. This is fivefold lower than the release probability in the resting state at $1.5 \mathrm{~mm}$ calcium, close to the in vivo concentration (Chanda and Xu-Friedman, 2010a). Large discrepancies in release probability between in vivo and in vitro data exist for other synapses, too (for review, see Borst, 2010). Possible explanations include tonic depression caused by spontaneous activity in vivo and differences in species, composition of extracellular fluid, or AP threshold.

Slice studies were performed in mice and rats. Their ultrasonic hearing results in poor phase locking of SBCs. In gerbils, the rostral AVCN, which contains the large SBCs, is much better developed than in mice (Gleich et al., 1998). Lacking comparative studies, we will assume that the basic transmission properties are conserved between large and small SBCs.

A higher AP threshold (e.g., caused by a lower input resistance in vivo) may explain the failures but not the lack of synaptic depression. Additional factors must be operating. The extracellular calcium concentration is lower in vivo than the $2 \mathrm{~mm}$ commonly used for slices, but, as discussed above, this difference is insufficient to explain the discrepancy. Importantly, the impact of inhibition is expected to be much weaker in slices where GABAergic neurons are generally not spontaneously active and the extracellular medium is continuously refreshed. The $\mathrm{GABA}_{\mathrm{B}}$ agonist baclofen blocks synaptic currents by $>90 \%$ in slices by activating presynaptic $\mathrm{GABA}_{\mathrm{B}}$ receptors on endbulbs (Chanda and Xu-Friedman, 2010b), yet baclofen hardly affects toneevoked responses in vivo (Ebert and Ostwald, 1995). The application of baclofen induced frequent postsynaptic AP failures and synaptic facilitation instead of depression in slices, similar to our in vivo observations (Chanda and Xu-Friedman, 2010b). We therefore hypothesize that $\mathrm{GABA}_{\mathrm{B}}$ receptors on the endbulb are 
tonically activated in vivo but not in slices. Postsynaptic $\mathrm{GABA}_{\mathrm{A}}$ or glycine receptors may also be more active, as suggested by the strong effects of $\mathrm{GABA}_{\mathrm{A}}$ and glycine antagonists on spontaneous firing by SBCs (Ebert and Ostwald, 1995; Kopp-Scheinpflug et al., 2002; Gai and Carney, 2008).

We address tonic depression as a final candidate. Most cells are silent in slices, but in vivo their spontaneous activity may induce tonic short-term synaptic depression (Boudreau and Ferster, 2005; Reig et al., 2006; Hermann et al., 2007; Wang et al., 2010). In slices, a fast recovery phase has been observed, which depends on calcium buildup within the endbulb (Wang and Manis, 2008; Yang and Xu-Friedman, 2008). Our data contained few long intervals caused by high spontaneous event rates, but the lack of recovery from depression even after intense stimulation (input rates $\gg 300$ spikes/s) is inconsistent with large tonic depression, assuming similar presynaptic calcium dynamics in vivo and in vitro. Our data thus suggest that, in vivo, the release probability of the endbulb of Held is sufficiently low to primarily evade synaptic depression. Instead, synaptic facilitation emerges, in agreement with slice studies (Bellingham and Walmsley, 1999; Oleskevich et al., 2000; Chanda and Xu-Friedman 2010b). Multiple mechanisms may contribute to transforming a safe but strongly depressing synapse observed in vitro into a synapse that tonically operates near AP threshold in vivo.

\section{Mechanisms of inhibition}

Synaptic inhibition is important in shaping temporal response patterns of SBCs (Kopp-Scheinpflug et al., 2002). We used juxtacellular recordings to quantify synaptic inhibition by monitoring its effect on the threshold EPSP, the minimal EPSP size that reliably triggers an AP. This extends previous work that used juxtacellular recordings to quantify both the strength of excitatory transmission and postsynaptic excitability (Lorteije et al., 2009). Major advantages of our approach over in vivo whole-cell or intracellular approaches are that it is technically much simpler and that no changes of the intracellular medium occur. Advantages over pharmacological approaches (Caspary et al., 1994; Kopp-Scheinpflug et al., 2002) and two-tone paradigms (Kopp-Scheinpflug et al., 2002) are the avoidance of network effects (e.g., disinhibition of excitatory inputs) and eliminating the need to disentangle cochlear suppression and synaptic inhibition (Kopp-Scheinpflug et al., 2002; Rhode and Greenberg, 1994) (T. Kuenzel, M. van der Heijden, J. G. G. Borst, unpublished results). A limitation of our method, however, is that changes in AP threshold can only be quantified if both subthreshold and suprathreshold EPSPs are present.

Our new approach allowed us to quantify both the spectral and temporal properties of synaptic inhibition over the entire response area. We observed a large overlap in tuning and strength of excitation and inhibition in SBCs, whereas thresholds of inhibition were generally higher than thresholds of excitation. Our data confirm previous work that inferred overlap of excitation and inhibition from nonlinear rate-level functions or the effects of blocking $\mathrm{GABA}_{\mathrm{A}}$ and glycine receptors (Caspary et al., 1994; Kopp-Scheinpflug et al., 2002). Kopp-Scheinpflug et al. (2002) also used waveform analysis to discriminate between presynaptic and postsynaptic events. Although their analysis may have started from an incorrect interpretation of complex waveforms (McLaughlin et al., 2008; Typlt et al., 2010) and did not consider spike depression or stochastic failures, many of our findings agree with theirs, including the delayed onset of inhibition. This delayed onset may appear surprising, given the proximity of the possible anatomical sources (Wickesberg and Oertel, 1990; Zhang and Oertel, 1993), but the slow time course of IPSPs in SBCs measured in vivo (Paolini and
Clark, 1998) and our model simulations suggest that the delayed effect of inhibition originates from a gradual summation of inhibitory conductance.

Our results indicate that inhibition acts by increasing the excitatory synaptic conductance needed to trigger a spike, in agreement with studies of other synapses (for review, see Silver, 2010). Our simulations suggest that a combination of hyperpolarizing and shunting inhibition is needed to explain the observed changes in extracellular waveform. Hyperpolarizing and shunting inhibition have similar effects on the input-output relationship (Silver, 2010), but the extra information provided by the waveform analysis allowed us to evaluate the relative impact of these mechanisms. Inhibition was accompanied by improvements in phase locking, in general agreement with previous results (Dehmel et al., 2010). This is surprising considering that EPSPs that barely exceed threshold introduce substantial jitter in EPSP-AP delay (Fig. 2b). A careful analysis, however, revealed that larger eEPSPs showed superior phase locking, possibly reflecting larger input convergence (Rothman et al., 1993; Joris et al., 1994; Xu-Friedman and Regehr, 2005) or, to a lesser degree, more synchronous vesicle release (Yang and Xu-Friedman, 2010). In the presence of inhibition, a larger fraction of spikes was triggered by these large, better-timed eEPSPs, effectively counteracting increased jitter caused by higher numbers of nearthreshold events.

\section{Function of inhibition}

An attractive property of synaptic inhibition is its capacity to create nonmonotonic rate-level relationships; other mechanisms such as synaptic depression cannot accomplish this efficiently. SBCs project to the MSO, which functions as a binaural coincidence detector (Grothe et al., 2010). Coincidence detectors can only work over a limited range of input conductances. Inhibition may keep the total input to MSO cells within this range, even when additional SBCs are recruited at higher intensities. The presence of rare MSO units having nonmonotonic monaural rate-level curves is consistent with this scheme (Goldberg and Brown, 1969; Grothe et al., 1997). Presumably, high spontaneous rates will hardly affect MSO, as convergent spontaneous inputs will not be synchronized. Spontaneous firing of SBCs may have a tonic effect on other SBC targets, as was shown for globular bushy cells (Kadner et al., 2006).

Considering that spontaneous firing indicates high sensitivity to inputs, inhibition accomplishes a shift in coding strategy: at low levels (without inhibition), SBCs are sensitive detectors that also encode stimulus intensity. At higher levels (with inhibition), sensitivity and rate code are lost in favor of a sparse, but accurate temporal coding of stimulus fine structure. Thus, synaptic inhibition allows SBCs to combine high sensitivity with a large dynamic range.

\section{References}

Azouz R, Gray CM (2000) Dynamic spike threshold reveals a mechanism for synaptic coincidence detection in cortical neurons in vivo. Proc Natl Acad Sci U S A 97:8110-8115.

Bazwinsky I, Härtig W, Rübsamen R (2008) Characterization of cochlear nucleus principal cells of Meriones unguiculatus and Monodelphis domestica by use of calcium-binding protein immunolabeling. J Chem Neuroanat 35:158-174.

Bellingham MC, Walmsley B (1999) A novel presynaptic inhibitory mechanism underlies paired pulse depression at a fast central synapse. Neuron 23:159-170.

Borst JGG (2010) The low synaptic release probability in vivo. Trends Neurosci 33:259-266 
Boudreau CE, Ferster D (2005) Short-term depression in thalamocortical synapses of cat primary visual cortex. J Neurosci 25:7179-7190.

Cant NB, Benson CG (2003) Parallel auditory pathways: projection patterns of the different neuronal populations in the dorsal and ventral cochlear nuclei. Brain Res Bull 60:457-474.

Cant NB, Morest DK (1979) The bushy cells in the anteroventral cochlear nucleus of the cat. A study with the electron microscope. Neuroscience 4:1925-1945.

Cant NB, Morest DK (1984) The structural basis for stimulus coding in the cochlear nucleus. In: Hearing sciences: recent advances (Berlin C, ed), pp 371-421. San Diego: College-Hill.

Cao XJ, Oertel D (2010) Auditory nerve fibers excite targets through synapses that vary in convergence, strength, and short-term plasticity. J Neurophysiol 104:2308-2320.

Cao XJ, Shatadal S, Oertel D (2007) Voltage-sensitive conductances of bushy cells of the mammalian ventral cochlear nucleus. Neurophysiology 97:3961-3975.

Caspary DM, Backoff PM, Finlayson PG, Palombi PS (1994) Inhibitory inputs modulate discharge rate within frequency receptive fields of anteroventral cochlear nucleus neurons. J Neurophysiol 72:2124-2133.

Chanda S, Xu-Friedman MA (2010a) A low-affinity antagonist reveals saturation and desensitization in mature synapses in the auditory brainstem. J Neurophysiol 103:1915-1926.

Chanda S, Xu-Friedman MA (2010b) Neuromodulation by GABA converts a relay into a coincidence detector. J Neurophysiol 104:2063-2074.

Dehmel S, Kopp-Scheinpflug C, Weick M, Dörrscheidt GJ, Rübsamen R (2010) Transmission of phase-coupling accuracy from the auditory nerve to spherical bushy cells in the Mongolian gerbil. Hear Res 268:234-249.

Ebert U, Ostwald J (1995) GABA can improve acoustic contrast in the rat ventral cochlear nucleus. Exp Brain Res 104:310-322.

Englitz B, Tolnai S, Typlt M, Jost J, Rübsamen R (2009) Reliability of synaptic transmission at the synapses of Held in vivo under acoustic stimulation. PLoS One 4:e7014.

Ferragamo MJ, Golding NL, Oertel D (1998) Synaptic inputs to stellate cells in the ventral cochlear nucleus. J Neurophysiol 79:51-63.

Frisina RD, Chamberlain SC, Brachman ML, Smith RL (1982) Anatomy and physiology of the gerbil cochlear nucleus: an improved surgical approach for microelectrode studies. Hear Res 6:259-275.

Gai Y, Carney LH (2008) Influence of inhibitory inputs on rate and timing of responses in the anteroventral cochlear nucleus. J Neurophysiol 99:1077-1095.

Gleich O, Kadow C, Strutz J (1998) The postnatal growth of cochlear nucleus subdivisions and neuronal somata of the anteroventral cochlear nucleus in the Mongolian gerbil (Meriones unguiculatus). Audiol Neurootol 3:1-20.

Goldberg JM, Brown PB (1969) Response of binaural neurons of dog superior olivary complex to dichotic tonal stimuli: some physiological mechanisms of sound localization. J Neurophysiol 32:613-636.

Grothe B, Park TJ, Schuller G (1997) Medial superior olive in the free-tailed bat: response to pure tones and amplitude-modulated tones. J Neurophysiol 77:1553-1565.

Grothe B, Pecka M, McAlpine D (2010) Mechanisms of sound localization in mammals. Physiol Rev 90:983-1012.

Guinan JJ, Li RY (1990) Signal processing in brainstem auditory neurons which receive giant endings (calyces of Held) in the medial nucleus of the trapezoid body of the cat. Hear Res 49:321-334.

Henze DA, Buzsáki G (2001) Action potential threshold of hippocampal pyramidal cells in vivo is increased by recent spiking activity. Neuroscience 105:121-130.

Hermann J, Pecka M, von Gersdorff H, Grothe B, Klug A (2007) Synaptic transmission at the calyx of Held under in vivo like activity levels. J Neurophysiol 98:807-820.

Hines ML, Carnevale NT (1997) The NEURON simulation environment. Neural Comput 9:1179-1209.

Howard MA, Burger RM, Rubel EW (2007) A developmental switch to GABAergic inhibition dependent on increases in Kv1-type $\mathrm{K}^{+}$currents. J Neurosci 27:2112-2123.

Isaacson JS, Walmsley B (1995) Counting quanta: direct measurements of transmitter release at a central synapse. Neuron 15:875-884.

Isaacson JS, Walmsley B (1996) Amplitude and time course of spontaneous and evoked excitatory postsynaptic currents in bushy cells of the anteroventral cochlear nucleus. J Neurophysiol 76:1566-1571.
Joris PX, Carney LH, Smith PH, Yin TC (1994a) Enhancement of neural synchronization in the anteroventral cochlear nucleus. I. Responses to tones at the characteristic frequency. J Neurophysiol 71:1022-1036.

Joris PX, Smith PH, Yin TC (1994b) Enhancement of neural synchronization in the anteroventral cochlear nucleus. II. Responses in the tuning curve tail. J Neurophysiol 71:1037-1051.

Juiz JM, Helfert RH, Bonneau JM, Wenthold RJ, Altschuler RA (1996) Three classes of inhibitory amino acid terminals in the cochlear nucleus of the guinea pig. J Comp Neurol 373:11-26.

Kadner A, Kulesza RJ, Berrebi AS (2006) Neurons in the medial nucleus of the trapezoid body and superior paraolivary nucleus of the rat may play a role in sound duration coding. J Neurophysiol 95:1499-1508.

Klepper A, Herbert H (1991) Distribution and origin of noradrenergic and serotonergic fibers in the cochlear nucleus and inferior colliculus of the rat. Brain Res 557:190-201.

Kopp-Scheinpflug C, Dehmel S, Dörrscheidt GJ, Rübsamen R (2002) Interaction of excitation and inhibition in anteroventral cochlear nucleus neurons that receive large endbulb synaptic endings. J Neurosci 22:11004-11018

Liberman MC (1991) Central projections of auditory-nerve fibers of differing spontaneous rate. I. Anteroventral cochlear nucleus. J Comp Neurol 313:240-258.

Lorteije JAM, Rusu SI, Kushmerick C, Borst JGG (2009) Reliability and precision of the mouse calyx of Held synapse. J Neurosci 29:13770-13784.

McLaughlin M, van der Heijden M, Joris PX (2008) How secure is in vivo synaptic transmission at the calyx of Held? J Neurosci 28:10206-10219.

Milenkovic I, Witte M, Turecek R, Heinrich M, Reinert T, Rübsamen R (2007) Development of chloride-mediated inhibition in neurons of the anteroventral cochlear nucleus of gerbil (Meriones unguiculatus). J Neurophysiol 98:1634-1644.

Oleskevich S, Clements J, Walmsley B (2000) Release probability modulates short-term plasticity at a rat giant terminal. J Physiol 524:513-523.

Osen KK (1969) Cytoarchitecture of the cochlear nuclei in the cat. J Comp Neurol 136:453-484.

Paolini AG, Clark GM (1998) Intracellular responses of the rat anteroventral cochlear nucleus to intracochlear electrical stimulation. Brain Res Bull 46:317-327.

Paolini AG, FitzGerald JV, Burkitt AN, Clark GM (2001) Temporal processing from the auditory nerve to the medial nucleus of the trapezoid body in the rat. Hear Res 159:101-116.

Pfeiffer RR (1966) Anteroventral cochlear nucleus: wave forms of extracellularly recorded spike potentials. Science 154:667-668.

Platkiewicz J, Brette R (2010) A threshold equation for action potential initiation. PLoS Comput Biol 6:e1000850.

Reig R, Gallego R, Nowak LG, Sanchez-Vives MV (2006) Impact of cortical network activity on short-term synaptic depression. Cereb Cortex 16:688-695.

Rhode WS, Greenberg S (1994) Lateral suppression and inhibition in the cochlear nucleus of the cat. J Neurophysiol 71:493-514.

Rodriguez-Molina VM, Aertsen A, Heck DH (2007) Spike timing and reliability in cortical pyramidal neurons: effects of EPSC kinetics, input synchronization and background noise on spike timing. PLoS One 2:e319.

Rothman JS, Manis PB (2003) The roles potassium currents play in regulating the electrical activity of ventral cochlear nucleus neurons. J Neurophysiol 89:3097-3113.

Rothman JS, Young ED, Manis PB (1993) Convergence of auditory nerve fibers onto bushy cells in the ventral cochlear nucleus: implications of a computational model. J Neurophysiol 70:2562-2583.

Ryugo DK, Sento S (1991) Synaptic connections of the auditory nerve in cats: relationship between endbulbs of held and spherical bushy cells. J Comp Neurol 305:35-48.

Schmiedt RA (1982) Boundaries of two-tone rate suppression of cochlearnerve activity. Hear Res 7:335-351.

Sento S, Ryugo DK (1989) Endbulbs of held and spherical bushy cells in cats: morphological correlates with physiological properties. J Comp Neurol 280:553-562.

Silver RA (2010) Neuronal arithmetic. Nat Rev Neurosci 11:474-489.

Typlt M, Haustein MD, Dietz B, Steinert JR, Witte M, Englitz B, Milenkovic I, Kopp-Scheinpflug C, Forsythe ID, Rübsamen R (2010) Presynaptic and postsynaptic origin of multicomponent extracellular waveforms at the endbulb of Held-spherical bushy cell synapse. Eur J Neurosci 31:1574-1581.

Varela JA, Sen K, Gibson J, Fost J, Abbott LF, Nelson SB (1997) A quantita- 
tive description of short-term plasticity at excitatory synapses in layer $2 / 3$ of rat primary visual cortex. J Neurosci 17:7926-7940.

Wang Y, Manis PB (2008) Short-term synaptic depression and recovery at the mature mammalian endbulb of Held synapse in mice. J Neurophysiol 100:1255-1264.

Wang Y, Ren C, Manis PB (2010) Endbulb synaptic depression within the range of presynaptic spontaneous firing and its impact on the firing reliability of cochlear nucleus bushy neurons. Hear Res 270:101-109.

Wickesberg RE, Oertel D (1988) Tonotopic projection from the dorsal to the anteroventral cochlear nucleus of mice. J Comp Neurol 268:389-399.

Wickesberg RE, Oertel D (1990) Delayed, frequency-specific inhibition in the cochlear nuclei of mice: a mechanism for monaural echo suppression. J Neurosci 10:1762-1768.

Winter IM, Palmer AR (1990) Responses of single units in the anteroventral cochlear nucleus of the guinea pig. Hear Res 44:161-178.

Wu SH, Oertel D (1986) Inhibitory circuitry in the ventral cochlear nucleus is probably mediated by glycine. J Neurosci 6:2691-2706.

Xu-Friedman MA, Regehr WG (2005a) Dynamic-clamp analysis of the ef- fects of convergence on spike timing. I. Many synaptic inputs. J Neurophysiol 94:2512-2525.

Xu-Friedman MA, Regehr WG (2005b) Dynamic-clamp analysis of the effects of convergence on spike timing. II. Few synaptic inputs. J Neurophysiol 94:2526-2534.

Yang H, Xu-Friedman MA (2008) Relative roles of different mechanisms of depression at the mouse endbulb of Held. J Neurophysiol 99:2510-2521.

Yang H, Xu-Friedman MA (2009) Impact of synaptic depression on spike timing at the endbulb of held. J Neurophysiol 102:1699-1710.

Yang H, Xu-Friedman MA (2010) Developmental mechanisms for suppressing the effects of delayed release at the endbulb of Held. J Neurosci 30:11466-11475.

Young ED, Sachs MB (2008) Auditory nerve inputs to cochlear nucleus neurons studied with cross-correlation. Neuroscience 154:127-138.

Zhang S, Oertel D (1993) Tuberculoventral cells of the dorsal cochlear nucleus of mice: intracellular recordings in slices. J Neurophysiol 69:1409_ 1421. 